\title{
ON THE SHAPE OF INTERLAYER VORTICES IN THE LAWRENCE-DONIACH MODEL
}

\author{
STAN ALAMA, LIA BRONSARD, AND ETIENNE SANDIER
}

\begin{abstract}
We consider the Lawrence-Doniach model for layered superconductors, in which stacks of parallel superconducting planes are coupled via the Josephson effect. To model experiments in which the superconductor is placed in an external magnetic field oriented parallel to the superconducting planes, we study the structure of isolated vortices for a doubly periodic problem. We consider a singular limit which simulates certain experimental regimes in which isolated vortices have been observed, corresponding to letting the interlayer spacing of the superconducting planes tend to zero and the Ginzburg-Landau parameter $\kappa \rightarrow \infty$ simultaneously, but at a fixed relative rate.
\end{abstract}

\section{INTRODUCTION}

Many high temperature superconductors are highly anisotropic crystalline materials composed of stacks of copper oxide superconducting planes separated by insulating or weakly superconducting material. Physicists have determined that an anisotropic Ginzburg-Landau model does not adequately describe the magnetic properties of such superconductors, and use instead a model introduced by Lawrence \& Doniach [LaDo] which properly captures the effect of the layered structure of these materials. These new effects are particularly noticable when an external "in-plane" field is applied to the sample, that is, the magnetic field is assumed to be parallel to the superconducting planes (see [KAVB], [ABeB1], [ABeB2]).

In 1998, Moler, Kirtley, Hinks, Li, \& Xu [Mo] published the results of an experiment on the high- $T_{c}$ material $\mathrm{Tl}_{2} \mathrm{Ba}_{2} \mathrm{CuO}_{6+\delta}$, determining the penetration depth $\lambda_{c}$ of the sample by measuring the apparent length along the direction of the planes of an isolated vortex produced by an in-plane applied field (an "interlayer vortex"). The calculation of $\lambda_{c}$ from the experimental data was done using a theory of interlayer vortices due to Clem \& Coffey [CmCo], in which a quasilinear equation describing the magnetic field profile is derived from physical principles. A similar equation was derived much earlier by Bulaevskii [Bu] starting from the LawrenceDoniach model. This equation features a "nonlinear core", due to the fact that the

Received by the editors March 8, 2004 and, in revised form, June 16, 2005.

2000 Mathematics Subject Classification. Primary 35J50, 58J37.

Key words and phrases. Calculus of variations, elliptic equations and systems, superconductivity, vortices.

The first and second authors were supported by an NSERC Research Grant. 
interaction between the layers is carried via the Josephson tunnelling effect, and linearizes to the usual London equation for the magnetic field when the field strength is small. As in the Clem \& Coffey paper, Moler et al. assume that the nonlinear effect of the core will be small at the scale of the experiment, and they fit their data to an (anisotropic) London model to arrive at their conclusion. The paper [Mo] was especially notable since it refuted the "interlayer tunnelling theory" of high- $T_{c}$ superconductors proposed by Anderson [An]. The issue became contentious when others, unsuccessfully attempting to solve Clem \& Coffey's quasilinear equation numerically, questioned its validity (see $[\mathrm{Fa}]$ ).

In this paper we consider isolated vortices for the Lawrence-Doniach model with an in-plane (parallel) applied field. We study a singular limit which mimics the experimental regime of Moler et al. and in fact is implicitly followed in the theoretical work by Clem \& Coffey and Bulaevskii, and derive the correct equations which describe the profiles of the magnetic field and order parameter near an interlayer vortex.

The Lawrence-Doniach Model. We assume that the superconducting planes are parallel to the $x z$ plane and that the magnetic field $\vec{h}$ in the sample is directed along the $z$-direction (parallel to the planes) and only depends on $x$ and $y$. In this way, the magnetic potential $\vec{A}$ defined by curl $\vec{A}=h$ only has two components:

$$
\begin{gathered}
\vec{h}=h(x, y) \hat{z} \\
\vec{A}=A_{x}(x, y) \hat{x}+A_{y}(x, y) \hat{y}, \quad \vec{h}=\operatorname{curl} \vec{A}=\left(\frac{\partial A_{y}}{\partial x}-\frac{\partial A_{x}}{\partial y}\right) \hat{z} .
\end{gathered}
$$

Since we are interested mainly in the shape of vortices, rather than imposing an external parallel field (in the $z$-direction) we consider a doubly periodic problem in the orthogonal plane. This avoids the problems of boundary conditions and the sticky relationship between the size of the applied field and the number of vortices present in a minimizer, and allows us to concentrate on finding the profile of the magnetic field near an isolated vortex. We assume the period domain $\Omega=$ $[-L, L] \times[-M, M]$ is a rectangle in $\mathbf{R}^{2}$ centered at the origin. We assume that there are $2 N$ superconducting planes separated by distance $s$ and located along the lines $y=\left(n-\frac{1}{2}\right) s, n=-N+1, \ldots, N$. (Note that this choice implies that the origin lies in a gap between the $n=0$ and $n=1$ planes, and $M=\left(N-\frac{1}{2}\right) s$.) One may think of this as describing an infinite, space-filling sample with periodic "vortex lattice", but it is also natural to think of $\Omega$ as a flat torus,

$$
\mathbf{T}:=\mathbf{R}^{2} /(2 L \mathbf{Z} \times 2 M \mathbf{Z})
$$

We also define one complex-valued order parameter $\psi_{n}$ per superconducting plane. Note that $\psi_{n}=\psi_{n}(x)$, since the superconductivity is assumed to reside in the copper-oxide planes and we assume uniformity in the $z$-direction. We choose nondimensional units in which the in-plane penetration depth $\lambda_{a}=1$. As usual, this introduces an important parameter, the Ginzburg-Landau parameter $\kappa$. In the Ginzburg-Landau model, $1 / \kappa$ represents the scale of variation for the superconducting order parameters, and in some sense measures the radius of the core region of an isolated vortex. For the high- $T_{c}$ superconductors it is large: $\kappa \simeq 100$ for $\mathrm{Tl}_{2} \mathrm{Ba}_{2} \mathrm{CuO}_{6+\delta}$ for example. 
Assuming that all gauge-invariant quantities are doubly periodic, it suffices to calculate the energy over a single period domain $\Omega$. The resulting LawrenceDoniach energy functional is then:

$$
\begin{aligned}
\mathcal{E}_{\kappa, s}^{L D}\left(\psi_{n}, A\right)=s & \sum_{n=1}^{N} \int_{-L}^{L}\left[\left|\left(\frac{d}{d x}-i A_{x}\left(x, y_{n}\right)\right) \psi_{n}\right|^{2}+\frac{\kappa^{2}}{2}\left(\left|\psi_{n}\right|^{2}-1\right)^{2}\right] d x \\
& +s \sum_{n=1}^{N} \int_{-L}^{L} \frac{1}{\lambda_{J}^{2} s^{2}}\left|\psi_{n}-\psi_{n-1} e^{i \int_{y_{n-1}}^{y_{n}} A_{y}(x, y) d y}\right|^{2} d x \\
& +\iint_{\Omega}\left(\frac{\partial A_{y}}{\partial x}-\frac{\partial A_{x}}{\partial y}\right)^{2} d x d y .
\end{aligned}
$$

The coupling between the superconducting planes given by the second sum in $\mathcal{E}_{\kappa, s}^{L D}$ simulates the Josephson effect, by which superconducting electrons travel from one superconducting region to another by quantum mechanical tunnelling. Finally, we note one further parameter, the Josephson penetration depth, $\lambda_{J}$, which measures the anisotropy between the in-plane conductivity and the interlayer (Josephson effect) conductivity. The content of the papers [ABeB1], [ABeB2] is the study of the limit $\lambda_{J} \rightarrow \infty$, and there are very interesting phenomena associated with this limit, in particular the "transparent state" predicted by Kes et al. [KAVB]. However, as explained in [ABeB1] this limit effectively describes behavior of a layered superconductor in a strong external parallel field, when the vortex lattice is very dense.

In this paper we fix $\lambda_{J}$ (which takes values near 200 in the experimental regime of [Mo]). In fact applying a change of variables to (1), $x \rightarrow \lambda_{J} x, A_{x} \rightarrow \frac{1}{\lambda_{J}} A_{x}$ (with $y, A_{y}$ unchanged) allows us to scale $\lambda_{J}$ out of the problem, and so we study the functional (1) with

$$
\lambda_{J}=1 \text {. }
$$

We note that this change of coordinates changes both the description of the fundamental domain $\left(L \rightarrow \frac{1}{\lambda_{J}} L\right)$ and the value of $\kappa\left(\kappa \rightarrow \lambda_{J} \kappa\right)$.

We pose the following mathematical problem, which we propose as a model for the experiments of Moler et al. We consider a limit which simultaneously sends $s \rightarrow 0$ and $\kappa \rightarrow \infty$ in such a way as to preserve the physically relevant regime $1 / \kappa \simeq<s$. More precisely, we assume that:

(H1) $\Omega, L, M$ are fixed with $L, M>>1, N \rightarrow \infty$ and $s=s_{N}=\frac{M}{N-\frac{1}{2}} \rightarrow 0$.

(H2) There exists a constant $\gamma>0$ such that $\kappa=\kappa_{N} \geq \gamma / s$.

We note that the formal calculations done by Clem \& Coffey [CmCo] and Bulaevskii $[\mathrm{Bu}]$ fit into this regime as well. We consider minimizers of the energy within a space of doubly periodic configurations with a fixed "degree", and study the asymptotic shape of minimizers. Our result (see Theorem 1.2 below) implies that isolated vortices have no "nonlinear core" in this limit, contrary to the predictions of $[\mathrm{Bu}, \mathrm{CmCo}]$.

We note that a different asymptotic regime, $s \rightarrow 0$ with $\kappa$ fixed, has been studied by Chapman, Du \& Gunzberger [ChDG] and by Bauman \& Ko [BaK].

Physical background. In their papers Bulaevskiı [Bu] and Clem \& Coffey [CmCo] both seek a continuum approximation for a semi-discrete model as the spacing $s \rightarrow 0$. In $[\mathrm{Bu}]$ the model is Lawrence-Doniach; in [CmCo] they start from an array 
of thin film superconductors coupled via the Josephson effect, but the arguments and final results are very similar. In both papers, the limit is never explicitly written, but finite differences in $n$ are approximated by derivatives in $y$. The other approximation made in both $[\mathrm{Bu}]$ and $[\mathrm{CmCo}]$ is that the magnitude of the order parameters $\left|\psi_{n}(x)\right|=1$ on each plane. This is explained on physical grounds by the idea that the core of the vortex will fit between adjacent planes, and therefore there is no need to lose condensation energy by supressing the value of the order parameter. Using these approximations and some additional physical reasoning, Bulaevskii arrives at the following equation to describe the magnetic field profile $h(x, y)$ near an isolated vortex:

$$
-\frac{\partial}{\partial x}\left[\arcsin \left(\lambda_{J}^{2} \frac{\partial h}{\partial x}\right)\right]-\frac{\partial^{2} h}{\partial y^{2}}+h=0
$$

expressed in our units. Clem \& Coffey arrive at a similar equation, again featuring the arcsin function, which arises because of the Josephson effect in which the tunneling currents in the $y$-direction are given in terms of the sine of the gaugeindependent phase difference across the two planes. Note that no singularity is prescribed on the right-hand side, but both authors assume a flux quantization condition which would suggest the presence of a term $2 \pi \delta(x)$ to represent the singular vortex core.

In the experimental regime of $[\mathrm{Mo}]$ the in-plane coherence length $\xi_{a}$ and the layer spacing are known to be of the same order of magnitude: in our units, this implies $s \simeq 1 / \kappa$. These are the smallest scales in the problem, and both are very small compared to the penetration depths $\lambda_{a}, \lambda_{J}$. Indeed, Clem \& Coffey explicitly state this relationship on the first page of their paper, and this is the motivation for our choice of the asymptotic regime defined by (H1), (H2).

Mathematical perspective. This problem brings several interesting mathematical points to resolve:

- How do we define a vortex? Since the order parameters $\psi_{n}(x)$ are only defined on the superconducting planes, there is no topological obstruction which forces them to vanish. As the physicists say, the cores lie in the gaps between the planes. Also, how do we define a "degree" for the discrete collection of functions $\psi_{n}(x)$ ? Rather than answer these points directly, we repose the question:

- How do we pose a variational problem which ensures the presence of vortices? Because nontrivial periodic fields and currents require nonperiodic vector potentials and order parameters, we use 't Hooft's boundary conditions, under which $\left(\psi_{n}, \vec{A}\right)$ are periodic up to a gauge transformation.

Definition 1.1. We say $\left(\psi_{n}, \vec{A}\right) \in t \mathcal{H}$ if $\psi_{n} \in H_{l o c}^{1}(\mathbf{R})$, for all $n \in \mathbf{Z}$, $\vec{A} \in H_{l o c}^{1}\left(\mathbf{R}^{2} ; \mathbf{R}^{2}\right)$, and there exist $\omega_{x}, \omega_{y} \in H_{l o c}^{2}\left(\mathbf{R}^{2}\right)$ such that

$$
\left\{\begin{array}{l}
\psi_{n}(x+2 L)=\psi_{n}(x) \exp \left\{i \omega_{x}(x, y)\right\}, \\
\psi_{n+2 N}(x)=\psi_{n}(x) \exp \left\{i \omega_{y}(x, y)\right\}, \\
\vec{A}(x+2 L, y)=\vec{A}(x, y)+\nabla \omega_{x}(x, y), \\
\vec{A}(x, y+2 M)=\vec{A}(x, y)+\nabla \omega_{y}(x, y) .
\end{array}\right.
$$


An immediate consequence of these conditions is the quantization of flux (see [ABeB1] for details): for any $\left(\psi_{n}, \vec{A}\right) \in t \mathcal{H}$ there exists $K \in \mathbf{Z}$ with

$$
\iint_{\Omega} \operatorname{curl} \vec{A} d x d y=2 \pi K \text {. }
$$

The magnetic potential $\vec{A}$ determines a connection 1 -form on the flat torus $\mathbf{T}$, the $\psi_{n}$ can be related to a section of a complex line bundle, and the Euler number gives the degree. Thus, the space of doubly periodic configurations splits into components determined by their degree $K$,

$$
t \mathcal{H}=\bigcup_{K \in \mathbf{Z}} t \mathcal{H}_{K} \quad \text { with } \quad t \mathcal{H}_{K}:=\left\{\left(\psi_{n}, \vec{A}\right) \in t \mathcal{H}: \iint_{\Omega} \operatorname{curl} \vec{A} d x d y=2 \pi K\right\},
$$

and we may minimize in any fixed class. This gives a roundabout answer to part of our first question: the degree is well-defined via the magnetic potential in the doubly periodic setting without regard to the order parameter. We discuss these issues in section 2.

- Does the energy determine the degree? The basic idea follows the seminal work of Bethuel, Brezis, \& Hélein $[\mathrm{BBH}]$ in identifying and isolating vortices for the Ginzburg-Landau functional. In section 3 we prove an upper bound on the energy in terms of the associated degree, which is on the order of $|\ln s|$. Section 4 is devoted to finding complementary lower bounds. The key to finding the lower bound is to extend the order parameters $\psi_{n}(x)$ to the gaps between the superconducting planes so we may have a quantity which is defined globally in $\Omega$. For $y_{n-1}<y \leq y_{n}$ we define the interpolation,

$$
\Psi(x, y):=\left[\frac{y-y_{n-1}}{s} \psi_{n}(x)+\frac{y_{n}-y}{s} \psi_{n-1}(x) e^{i \int_{y_{n-1}}^{y_{n}} A_{y}(x, y) d y}\right] e^{-i \int_{y}^{y_{n}} A_{y}(x, u) d u} .
$$

Then $\Psi(x, y)$ extends the order parameters to all of $\mathbf{R}^{2}$ with 't Hooft-type boundary conditions. The "vortices" are then defined by energy concentration, using the vortex-ball construction of Sandier [S]. (Similar methods have also been introduced by Jerrard [Je].) In fact, in this paper by "vortices" we mean "vortex balls", that is, a finite collection of small disks in which the energy concentrates at the order $|\ln s|$. This definition allows us to distinguish point vortices in the limit, but leaves unanswerable the question above as to their exact location relative to the superconducting planes for small $s>0$, as the radius of the vortex balls will be large compared to $s$. This analysis will be done in section 4 .

The lower bounds and vortex-ball construction also respond to a question neglected in the physicists' work: what is the form of the singularity at the core? The treatments of $[\mathrm{Bu}],[\mathrm{CmCo}]$ derive equations (such as (2)) for the magnetic field away from the singular core, avoiding the distributional limit of the right-hand side at the singularity due to the vortex core. It was not clear from the experiments that the vortex core could not have been a line segment, or that its contribution to the limiting equation could not have been a more complicated distribution.

- What is the shape of the limiting order parameter and field $h$ ? In section 5 we pass to the limit in the exterior of the balls and find the limiting equations for the order parameters and magnetic field. The Euler-Lagrange 
equations for $\psi_{n}, h$ are of mixed differential and finite-difference types, and lacking the usual elliptic regularity theory for these equations we only obtain relatively weak convergence for the minimizers.

We prove the following result:

Theorem 1.2. Let $\left(\psi_{n}^{s}, \vec{A}^{s}\right)$ attain $\inf _{t \mathcal{H}_{K}} \mathcal{E}_{\kappa, s}^{L D}$, and let $\Psi_{s}=\Psi(x, y)$ defined as in (5) for each $s$. Then there exists a subsequence $s \rightarrow 0$, a finite set of points $V:=\left\{a_{1}, \ldots, a_{K}\right\} \in \Omega$ and $\Psi_{0} \in H_{l o c}^{1}(\Omega \backslash V), \vec{A}^{0} \in H^{1}(\Omega)$ such that as $s \rightarrow 0$ :

$$
\begin{aligned}
& \Psi_{s} \rightarrow \Psi_{0} \quad \text { weakly in } H_{l o c}^{1}(\Omega \backslash V), \\
& \vec{A}^{s} \rightarrow \vec{A}^{0}, \quad \text { weakly in } H^{1}(\Omega), \text { and }
\end{aligned}
$$

(a) $\Psi_{0}=1$ almost everywhere in $\Omega$ and $\Psi_{0}$ is the canonical harmonic map $\Omega \backslash V \rightarrow S^{1}$, with degree one at each defect $a_{i}, i=1, \ldots, K$.

(b) $h_{0}(x, y):=\operatorname{curl} \vec{A}^{0}(x, y) \in L^{2}(\Omega)$ solves

$$
\left\{\begin{array}{l}
-\Delta h_{0}+h_{0}=2 \pi \sum_{i=1}^{K} \delta_{a_{i}}(x, y) \quad \text { for }(x, y) \in \Omega, \\
h_{0}(x+2 L, y)=h_{0}(x, y)=h_{0}(x, y+2 M) \quad \text { for }(x, y) \in \mathbf{R}^{2},
\end{array}\right.
$$

in the sense of distributions in $\Omega$.

(c) The minimal energy satisfies

$$
\mathcal{E}_{\kappa, s}^{L D}\left(\psi_{n}^{s}, \vec{A}^{s}\right)=2 \pi K|\log s|+O(1),
$$

as $s \rightarrow 0$.

In the original units (with $\lambda_{J}$ ) and in the case of a single isolated vortex $K=1$, the magnetic field profile converges to an anisotropic London equation,

$$
-\lambda_{J}^{2} \partial_{x}^{2} h-\partial_{y}^{2} h+h=2 \pi \delta_{0} .
$$

In particular we find that there is no "nonlinear" effect at the core of a vortex as predicted by the physicists $[\mathrm{Bu}],[\mathrm{CmCo}]$, at least not in this asymptotic limit. Contrary to these papers, when $s$ is small and $\kappa \simeq 1 / s$ is large, at the scale of the penetration depth an interlayer vortex in the Lawrence-Doniach model simply resembles a vortex for the corresponding anisotropic Ginzburg-Landau model. From our analysis, the conclusions drawn by Moler et al. [Mo], based on their experimental results fitted to the anisotropic London model, are completely consistent with the Lawrence-Doniach model.

Remark 1.3. Our results apply to the case where an external magnetic field is present as follows.

Consider the modified energy functional $\mathcal{E}_{\kappa, s, H}^{L D}$ obtained from $\mathcal{E}_{\kappa, s}^{L D}$ by replacing the last integral in (1) by

$$
\iint_{\Omega}\left(\frac{\partial A_{y}}{\partial x}-\frac{\partial A_{x}}{\partial y}-H\right)^{2} d x d y
$$

where $H>0$ represents the magnitude of an applied magnetic field parallel to the planes. Recall that we are in the periodic setting, hence we work in the periodic cell $\Omega$. Then if $\left(\psi_{n}, \vec{A}\right) \in t \mathcal{H}_{K}$ we deduce from (4) that

$$
\mathcal{E}_{\kappa, s, H}^{L D}\left(\psi_{n}, \vec{A}\right)=\mathcal{E}_{\kappa, s}^{L D}\left(\psi_{n}, \vec{A}\right)-4 \pi K H+|\Omega| H^{2} .
$$


Thus using item (c) in the above theorem we find

$$
\min _{t \mathcal{H}_{K}} \mathcal{E}_{\kappa, s, H}^{L D}=2 \pi K(|\log s|-2 H)+|\Omega| H^{2}+O(1),
$$

as $s \rightarrow 0$.

In particular if $C$ is large enough and if $H<\frac{1}{2}|\log s|-C$, the optimal value of $K$ is 0 for any small enough $s$, whereas if $H>\frac{1}{2}|\log s|+C$ the optimal value of $K$ will be nonzero. We therefore recover the classical value of the first critical field computed by Abrikosov in the framework of the Ginzburg-Landau model, but here $1 / s$ plays the role of the Ginzburg-Landau parameter $\kappa$. Note that $1 / s$, just as $\kappa$, is the ratio of two lengths: the distance between the layers divided by the in-plane penetration depth.

\section{VARIATIONAL FORMULATION}

We consider a periodic problem on a rectangular lattice with fundamental domain

$$
\Omega:=[-L, L] \times[-M, M] .
$$

We could just as well study other period geometries (for example with an equilateral triangular lattice, as is observed for type-II superconductors), and the result would be identical. We assume that the period domain $\Omega$ contains $2 N$ superconducting planes $\Sigma_{n}(n=1-N, \ldots, N)$ with separation distance $s$, at locations

$$
y_{n}:=\left(n-\frac{1}{2}\right) s, \quad n=1-N, \ldots, N .
$$

On each plane is defined a complex order parameter $\psi_{n}(x) \in \mathbf{C}$, and a vector potential $\vec{A}(x, y)$ is defined on all of $\mathbf{R}^{2}$.

We suppose that all gauge-invariant quantities are doubly periodic, and therefore it suffices to calculate the energy over a single period domain $\Omega$,

$$
\begin{aligned}
\mathcal{E}_{\kappa, s}^{L D}\left(\psi_{n}, \vec{A}\right)=s & \sum_{n=1}^{N} \int_{-L}^{L}\left[\left|\left(\frac{d}{d x}-i A_{x}\left(x, y_{n}\right)\right) \psi_{n}\right|^{2}+\frac{\kappa^{2}}{2}\left(\left|\psi_{n}\right|^{2}-1\right)^{2}\right] d x \\
& +s \sum_{n=1}^{N} \int_{-L}^{L} \frac{1}{s^{2}}\left|\psi_{n}-\psi_{n-1} e^{i \int_{y_{n-1}}^{y_{n}} A_{y}(x, y) d y}\right|^{2} d x \\
& +\iint_{\Omega}\left(\frac{\partial A_{y}}{\partial x}-\frac{\partial A_{x}}{\partial y}\right)^{2} d x d y .
\end{aligned}
$$

Note that $\mathcal{E}_{\kappa, s}^{L D}$ is expressed in nondimensional units, chosen such that the in-plane penetration depth $\lambda_{a b}=1, \kappa=\lambda_{a b} / \xi_{a b}$ is the Ginzburg Landau parameter. We have also chosen to scale distances in $x$ to eliminate the Josephson penetration depth $\lambda_{J}$ appearing in the coupling term.

The spaces $t \mathcal{H}_{K}$ introduced in Definition 1.1 are unwieldy for analysis, both for the usual reason of gauge invariance, a noncompact symmetry action, and because of the complicated nature of the 't Hooft condition itself. Fortunately, it is possible to fix a gauge to solve both problems at once. 
Definition 2.1. We say $\left(\psi_{n}, \vec{A}\right) \in t \mathcal{H}_{K}^{*}$ if $\psi_{n} \in H_{l o c}^{1}(\mathbf{R})$, for all $n \in \mathbf{Z}, \vec{A} \in$ $H_{l o c}^{1}\left(\mathbf{R}^{2} ; \mathbf{R}^{2}\right)$, and there exist constants $\omega_{1}, \omega_{2} \in \mathbf{R}$ and $\xi \in H_{l o c}^{2}\left(\mathbf{R}^{2}\right)$ such that

$$
\left\{\begin{array}{l}
\psi_{n}(x+2 L)=\psi_{n}(x) \exp \left\{i \omega_{1}\right\} \\
\psi_{n+2 N}(x)=\psi_{n}(x) \exp \left\{i\left(\omega_{2}-\frac{K \pi}{L} x\right)\right\} \\
\vec{A}(x, y)=\frac{K \pi}{2 L M}(-y, 0)+\nabla^{\perp} \xi(x, y) \\
\xi(x+2 L, y)=\xi(x, y)=\xi(x, y+2 M) .
\end{array}\right.
$$

Note that with this definition, $\operatorname{div} \vec{A}(x, y)=0$ and

$$
\begin{aligned}
& \vec{A}(x+2 L, y)=\vec{A}(x, y), \\
& \vec{A}(x, y+2 M)=\vec{A}(x, y)+\left(\frac{K \pi}{L}, 0\right) .
\end{aligned}
$$

This choice is a "Coulomb gauge", in the sense that the energy controls the $H^{1}$ norm of $\vec{A}$ :

Lemma 2.2. (a) For every $\left(\psi_{n}, \vec{A}\right) \in t \mathcal{H}_{K}$ there exists $\lambda \in H_{\text {loc }}^{2}\left(\mathbf{R}^{2}\right)$ such that

$$
\begin{gathered}
\left(\psi_{n} \exp \left\{-i \lambda\left(x, y_{n}\right)\right\}, \vec{A}-\nabla \lambda\right) \in t \mathcal{H}_{K}^{*}, \quad \text { and } \\
\mathcal{E}_{\kappa, s}^{L D}\left(\psi_{n} \exp \left\{-i \lambda\left(x, y_{n}\right)\right\}, \vec{A}-\nabla \lambda\right)=\mathcal{E}_{\kappa, s}^{L D}\left(\psi_{n}, \vec{A}\right) .
\end{gathered}
$$

(b) There exists $C_{0}$ such that for all $\left(\psi_{n}, \vec{A}\right) \in t \mathcal{H}_{K}^{*}$,

$$
\|\vec{A}\|_{H^{1}(\Omega)}^{2} \leq C_{0}\|\operatorname{curl} \vec{A}\|_{L^{2}(\Omega)}^{2} .
$$

The proof of Lemma 2.2 is contained in [ABeB1].

The Euler-Lagrange equations under 't Hooft conditions are obtained by varying the energy with respect to smooth doubly-periodic test functions. We obtain:

$$
\begin{aligned}
& 0=-\left(\partial_{x}-i A_{x}\left(x, y_{n}\right)\right)^{2} \psi_{n}+\kappa^{2}\left(\left|\psi_{n}\right|^{2}-1\right) \psi_{n} \\
& +\frac{1}{s^{2}}\left[2 \psi_{n}-\psi_{n+1} e^{-i \int_{y_{n}}^{y_{n}+1} A_{y}(x, y) d y}-\psi_{n-1} e^{i \int_{y_{n-1}}^{y_{n}} A_{y}(x, y) d y}\right] \text {; } \\
& \frac{\partial h}{\partial y}(x, y)=0, \quad y \neq y_{n} \\
& h\left(x, y_{n}+\right)-h\left(x, y_{n}-\right)=s j_{x}^{(n)}:=s \operatorname{Im}\left\{\psi_{n}^{*}\left(\partial_{x}-i A_{x}\left(x, y_{n}\right)\right) \psi_{n}\right\} ; \\
& \frac{\partial h}{\partial x}=-j_{y}^{(n)}:=-\frac{1}{s} \operatorname{Im}\left\{\psi_{n}^{*}\left(\psi_{n}-\psi_{n-1} e^{i \int_{y_{n-1}}^{y_{n}} A_{y} d y}\right)\right\} \\
& =\frac{1}{s} \operatorname{Im}\left\{\psi_{n}^{*} \psi_{n-1} e^{i \int_{y_{n-1}}^{y_{n}} A_{y}(x, y) d y}\right\}, \text { for } y_{n-1}<y<y_{n} .
\end{aligned}
$$

In light of equation (9) we define

$$
h^{(n)}(x):=h(x, y) \text { for } y_{n-1}<y<y_{n} .
$$

In particular, (10) implies a finite-difference equation for $h^{(n)}$ :

$$
\frac{h^{(n+1)}(x)-h^{(n)}(x)}{s}=j_{x}^{(n)}, \quad n \in \mathbf{Z} .
$$

For later purposes, we also record the current conservation equation

$$
\partial_{x} j_{x}^{(n)}(x)+\frac{1}{s}\left(j_{y}^{(n+1)}-j_{y}^{(n)}\right)=0 .
$$


This equation is not independent of the others: it can be derived by differentiating (10) and substituting into (11). This is not surprising since $\phi_{n}$ and $\vec{A}$ are related through gauge invariance. We note that $j_{x}^{(n)}(x)$ measures the current density within the $n^{\text {th }}$ superconducting plane, while $j_{y}^{(n)}(x)$ gives the Josephson current density in the gap between the $(n-1)^{s t}$ and $n^{t h}$ planes. In this way we may view (13) as a semi-discrete version of the classical equation of continuity $\operatorname{div} \vec{j}=0$. This is the conservation law corresponding to the $U(1)$ gauge invariance as guaranteed by Noether's Theorem.

We have the following basic consequence of the Maximum Principle:

Lemma 2.3. Suppose $\left(\psi_{n}, \vec{A}\right) \in t \mathcal{H}$ is a solution of the Euler-Lagrange equations (8)-(11). Then $\left|\psi_{n}(x)\right| \leq 1$ for all $x \in \mathbf{R}$ and $n \in \mathbf{Z}$.

Proof. We calculate:

$$
\begin{aligned}
\frac{1}{2} \partial_{x}^{2}\left(\left|\psi_{n}\right|^{2}\right)= & \operatorname{Re}\left\{\psi_{n}^{*}\left(\partial_{x}-i A_{x}\left(x, y_{n}\right)\right)^{2} \psi_{n}\right\}+\left|\left(\partial_{x}-i A_{x}\left(x, y_{n}\right)\right) \psi_{n}\right|^{2} \\
= & \kappa^{2}\left(\left|\psi_{n}\right|^{2}-1\right)\left|\psi_{n}\right|^{2}+\left|\left(\partial_{x}-i A_{x}\left(x, y_{n}\right)\right) \psi_{n}\right|^{2} \\
& +\frac{1}{s} \operatorname{Re}\left\{2\left|\psi_{n}\right|^{2}-\psi_{n}^{*} \psi_{n+1} e^{-i \int_{y_{n}}^{y_{n}+1} A_{y} d y}-\psi_{n}^{*} \psi_{n-1} e^{i \int_{y_{n}-1}^{y_{n}} A_{y} d y}\right\}
\end{aligned}
$$

Assume

$$
\max _{k, x}\left|\psi_{k}(x)\right|=\left|\psi_{n}\left(x_{0}\right)\right|>1 .
$$

Then $\partial_{x}^{2}\left(\left|\psi_{n}\right|^{2}\right)\left(x_{0}\right) \leq 0,\left(\left|\psi_{n}\left(x_{0}\right)\right|^{2}-1\right)\left|\psi_{n}\left(x_{0}\right)\right|^{2}>0$, and

$$
\begin{aligned}
& \operatorname{Re}\left\{\psi_{n}^{*} \psi_{n+1} e^{-i \int_{y_{n}}^{y_{n}+1} A_{y} d y}\right\} \leq\left|\psi_{n}\right|\left|\psi_{n+1}\right| \leq\left|\psi_{n}\right|^{2}\left(x_{0}\right), \\
& \operatorname{Re}\left\{\psi_{n}^{*} \psi_{n-1} e^{i \int_{y_{n-1}}^{y_{n}} A_{y} d y}\right\} \leq\left|\psi_{n}\right|\left|\psi_{n-1}\right| \leq\left|\psi_{n}\right|^{2}\left(x_{0}\right) .
\end{aligned}
$$

But this contradicts the computation above.

\section{UPPER BOUNDS ON THE ENERGY}

We fix the vortex number $K \geq 1$ and consider a limit which simultaneously sends $s \rightarrow 0$ and $\kappa \rightarrow \infty$ in such a way as to preserve the physically relevant regime $\xi_{a b} \lesssim s$. More precisely, we assume that:

(H1) $\Omega, L, M$ are fixed with $L, M>>1, N \rightarrow \infty$ and $s=s_{N}=\frac{M}{N-\frac{1}{2}} \rightarrow 0$.

(H2) There exists a constant $\gamma>0$ such that $\kappa \geq \gamma / s$.

We prove the following upper bound:

Proposition 3.1. For any $K \in \mathbf{Z}, K \geq 1$, there exists a constant $C$ independent of $\kappa$, s such that

$$
\inf _{\left(\psi_{n}, \vec{A}\right) \in t \mathcal{H}_{K}^{*}} \mathcal{E}_{\kappa, s}^{L D}\left(\psi_{n}, \vec{A}\right) \leq 2 \pi K|\ln s|+C .
$$

We begin with the case $K=1$ and sketch the modifications required to prove the general case at the end of the section.

First, define a smooth cut-off function, $\zeta \in C^{\infty}([0, \infty))$ with $0 \leq \zeta(t) \leq 1$ for all $t \in[0, \infty), \zeta(t)=1$ for $t \geq 1$ and $\zeta(t)=0$ for $t \leq \frac{1}{2}$. For $\mathbf{x}:=(x, y) \in$ 
$[1-L, L-1] \times[1-M, M-1]$ we let

$$
\Psi(\mathbf{x})=\tilde{\Psi}(\mathbf{x}):=\frac{\mathbf{x}}{|\mathbf{x}|} \zeta\left(2 \frac{|\mathbf{x}|}{s}\right) .
$$

Near the edges of the period rectangle $\Omega$ we must correct $\Psi$ to satisfy the 't Hooft conditions (7). In particular we may extend $\Psi$ to be piecewise smooth in $\Omega=$ $[-L, L] \times[M, M]$ by choosing a smooth function in the annular region

$$
\mathcal{A}=\Omega \backslash[1-L, L-1] \times[1-M, M-1]
$$

such that $|\Psi(\mathbf{x})| \equiv 1$ in $\mathcal{A}$ and on $\partial \Omega$,

$$
\Psi(\mathbf{x})= \begin{cases}1, & \text { when } x=L,-M \leq y \leq M \\ -1, & \text { when } x=-L,-M \leq y \leq M \\ e^{ \pm i \pi \frac{L-x}{2 L}}, & \text { when } y= \pm M,-L \leq x \leq L .\end{cases}
$$

The existence of such an extension is guaranteed by the fact that the degree is exactly one on each bounding rectangle. (In fact the function may be constructed explicitly by linearly interpolating the phase in the annular region.) The resulting function $\Psi(\mathbf{x})$ is Lipshitz in $\Omega$ and $|\Psi(\mathbf{x})|=1$ in $\Omega \backslash B_{s / 2}(0)$. Following Bethuel \& Rivière $[\mathrm{BeRi}]$ we also define $\vec{A}$ via:

$$
\vec{A}(x, y)=\left(\operatorname{Im}\left(\Psi^{*} \partial_{x} \Psi\right), \operatorname{Im}\left(\Psi^{*} \partial_{y} \Psi\right)\right) \zeta\left(\frac{|\mathbf{x}|}{2}\right) .
$$

With this definition we verify that

$$
\operatorname{curl} \vec{A}=0, \quad(\nabla-i \vec{A}) \Psi=0 \quad \text { for all } \mathbf{x} \in \Omega \backslash B_{2}(0) .
$$

We then extend $\Psi, \vec{A}$ to all of $\mathbf{R}$ via 't Hooft conditions. First note that by construction

$$
\begin{aligned}
& \Psi(L, y)=\Psi(-L, y) \exp (i \pi), \vec{A}(L, y)=\vec{A}(-L, y), \quad-M \leq y \leq M \\
& \Psi(x, M)=\Psi(x,-M) \exp \left(i \pi\left[1-\frac{x}{L}\right]\right), \vec{A}(x, M)=\vec{A}(x,-M)-\frac{\pi}{L},-L \leq x \leq L .
\end{aligned}
$$

Hence we can define $\Psi, \vec{A}$ globally using the periodicity conditions

$$
\begin{aligned}
& \Psi(x+2 L, y)=\Psi(x, y) \exp (i \pi), \vec{A}(x+2 L, y)=\vec{A}(x, y) \\
& \Psi(x, y+2 M)=\Psi(x, y) \exp \left(i \pi\left[1-\frac{x}{L}\right]\right), \vec{A}(x, y+2 M)=\vec{A}(x, y)-\frac{\pi}{L}
\end{aligned}
$$

Finally we set

$$
\psi_{n}(x)=\Psi\left(x, y_{n}\right), \quad x \in[-L, L], \quad n \in \mathbf{Z} .
$$

Note that $\left|\psi_{n}(x)\right|=1$ for all $n$ and for all $x \in[-L, L]$, and (17), (18) imply that $\left(\psi_{n}, \vec{A}\right) \in t \mathcal{H}_{1}$. It is not necessarily true that $\left(\psi_{n}, \vec{A}\right) \in t \mathcal{H}_{1}^{*}$, but by Lemma 2.2 it is gauge equivalent to an element of $t \mathcal{H}_{1}^{*}$, and the value of $\mathcal{E}_{\kappa, s}^{L D}$ is exactly the same.

To estimate $\inf _{t \mathcal{H}_{1}^{*}} \mathcal{E}_{\kappa, s}^{L D}$ we must carefully calculate the energy of this configuration. First, by (16)

$$
\iint_{\Omega}(\operatorname{curl} \vec{A})^{2} d x d y=\iint_{B_{2}(0)}(\operatorname{curl} \vec{A})^{2} d x d y=C_{1},
$$

independent of $s, \kappa$. Also by (16), we have for all $(x, y) \in \Omega \backslash B_{2}(0)$,

$$
\partial_{x} \psi_{n}-i A_{x}\left(x, y_{n}\right) \psi_{n}=\partial_{x} \Psi\left(x, y_{n}\right)-i A_{x}\left(x, y_{n}\right) \Psi\left(x, y_{n}\right)=0
$$


and

$$
\begin{aligned}
\psi_{n}-\psi_{n-1} e^{i \int_{y_{n-1}}^{y_{n}} A_{y}(x, y) d y} & =\int_{y_{n-1}}^{y_{n}} \partial_{y}\left[\Psi(x, y) e^{i \int_{y}^{y_{n}} A_{y}\left(x, y^{\prime}\right) d y^{\prime}}\right] d y \\
& =\int_{y_{n-1}}^{y_{n}}\left[\partial_{y} \Psi-i A_{y}(x, y) \Psi\right] e^{i \int_{y}^{y_{n}} A_{y}\left(x, y^{\prime}\right) d y^{\prime}} d y=0 .
\end{aligned}
$$

Thus all contributions to the energy comes from $B_{2}(0) \subset[-2,2]^{2}$. Let $\tilde{N}=\tilde{N}(s):=$ $\left[\frac{2}{s}+\frac{1}{2}\right]$, so that $\left|y_{n}\right| \leq 2$ if and only if $\tilde{N}-1 \leq n \leq \tilde{N}$. We have that:

$$
\mathcal{E}_{\kappa, s}^{L D}\left(\psi_{n}, \vec{A}\right)=s \sum_{n=1-\tilde{N}}^{\tilde{N}} \int_{-2}^{2}\left\{\left|\psi_{n}^{\prime}\right|^{2}+\frac{1}{s^{2}}\left|\psi_{n}-\psi_{n-1}\right|^{2}\right\} d x+O(1) .
$$

Now, for $1-\tilde{N} \leq n \leq \tilde{N}$,

$$
\begin{aligned}
\frac{1}{s} \sum_{n=1-\tilde{N}}^{\tilde{N}} \int_{-2}^{2}\left|\psi_{n}-\psi_{n-1}\right|^{2} d x & =\frac{1}{s} \sum_{n=1-\tilde{N}}^{\tilde{N}} \int_{-2}^{2}\left|\int_{y_{n-1}}^{y_{n}} \partial_{y} \Psi d y\right|^{2} d x \\
& \leq \iint_{[-2,2]^{2}}\left|\partial_{y} \Psi\right|^{2} d x d y \\
& \leq \iint_{[-2,2]^{2} \backslash B_{s / 2}}\left|\partial_{y}\left(\frac{\mathbf{x}}{|\mathbf{x}|}\right)\right|^{2} d x d y \\
& \quad+\iint_{B_{s / 2} \backslash B_{s / 4}}\left|\partial_{y}\left(\frac{\mathbf{x}}{|\mathbf{x}|} \zeta(2|\mathbf{x}| / s)\right)\right|^{2} d x d y \\
& \leq \pi \ln \left(\frac{4}{s}\right)+2 \pi \int_{s / 4}^{s / 2}\left(\frac{1}{r^{2}}+\frac{C}{s^{2}}\right) r d r \\
& =\pi|\ln s|+O(1),
\end{aligned}
$$

where we denote by $O(1)$ quantities bounded independent of $s, \kappa$.

Next, we calculate:

$$
\begin{aligned}
\int_{-2}^{2}\left|\psi_{n}^{\prime}\right|^{2} d x & =\int_{-2}^{2} \frac{y_{n}^{2}}{\left(x^{2}+y_{n}^{2}\right)} d x \\
& =\frac{1}{1+y_{n}^{2}}+\frac{1}{y_{n}} \arctan \left(\frac{1}{y_{n}}\right) .
\end{aligned}
$$

We have the following estimates:

$$
\begin{aligned}
& s \sum_{n=1-\tilde{N}}^{\tilde{N}} \frac{1}{1+y_{n}^{2}} \leq \int_{-1}^{1} \frac{1}{1+y^{2}} d y \leq 2 \pi \\
& s \sum_{n=1}^{\tilde{N}} \frac{1}{y_{n}} \arctan \left(\frac{1}{y_{n}}\right) \leq \frac{\pi}{2} s \sum_{n=1}^{\tilde{N}} \frac{1}{y_{n}}=\frac{\pi}{2} \int_{s / 2}^{y_{\tilde{N}}} \frac{d y}{y}+O(1)=\frac{\pi}{2}|\ln s|+O(1) ; \\
& s \sum_{n=1-\tilde{N}}^{0} \frac{1}{y_{n}} \arctan \left(\frac{1}{y_{n}}\right) \leq \frac{\pi}{2}|\ln s|+O(1) .
\end{aligned}
$$


Together we obtain

$$
\int_{-2}^{2}\left|\psi_{n}^{\prime}\right|^{2} d x=\pi|\ln s|+O(1)
$$

and combining with (20) and (19) we have the desired upper bound for the case $K=1$,

$$
\inf _{t \mathcal{H}_{1}^{*}} \mathcal{E}_{\kappa, s}^{L D} \leq \mathcal{E}_{\kappa, s}^{L D}\left(\psi_{n}, \vec{A}\right) \leq 2 \pi|\ln s|+O(1),
$$

with $O(1)$ term independent of $s, \kappa$.

To complete the case of general $K \geq 2$, we construct $\left(\psi_{n}, \vec{A}\right)$ as above for the period domain $\Omega^{\prime}=[-L / K, L / K] \times[-M, M]$. Then on the larger domain $\Omega=$ $[-L, L] \times[-M, M]$ we have an admissible configuration in $t \mathcal{H}_{K}$, whose energy in $\Omega$ is exactly $K$ times the energy calculated in $\Omega^{\prime}$. This completes the proof of Propositon 3.1. $\vec{j}^{(n)}$.

We conclude this section with some basic estimates on the field $h$ and currents

Lemma 3.2. Suppose $\left(\psi_{n}, \vec{A}\right) \in t \mathcal{H}_{K}^{*}$ is a critical point of $\mathcal{E}_{\kappa, s}^{L D}$ with

$$
\mathcal{E}_{\kappa, s}^{L D}\left(\psi_{n}, \vec{A}\right) \leq C_{0}|\ln s|
$$

for a fixed constant $C_{0}$ independent of $s, \kappa$. Then

$$
\begin{aligned}
\left|j_{x}^{(n)}(x)\right| & =\left|\operatorname{Im}\left(\psi_{n}^{*}\left(\partial_{x}-i A_{x}\left(x, y_{n}\right)\right) \psi_{n}\right)\right| \leq \frac{3}{s}|\ln s|, \\
\|h\|_{L^{\infty}} & \leq \frac{2}{s}|\ln s| .
\end{aligned}
$$

Proof. First we claim that for every $n$ and for every interval $I$ with length at least $s$ there exists $x_{0} \in I$ with

$$
\left|j_{x}^{(n)}\left(x_{0}\right)\right| \leq \frac{1}{s}|\ln s|
$$

Indeed, suppose the contrary: there exists $n_{0}$ and an interval $I_{0}$ with $\left|I_{0}\right| \geq s$ and $\left|j_{x}^{(n)}(x)\right| \geq \frac{1}{s}|\ln s|$ for all $x \in I_{0}$. Then

$$
\begin{aligned}
C_{0}|\ln s| & \geq s \sum_{n=1-N}^{N} \int_{-L}^{L}\left|\left(\partial_{x}-i A_{x}\left(x, y_{n}\right)\right) \psi_{n}\right|^{2} d x \\
& \geq s \int_{I_{0}}\left|\left(\partial_{x}-i A_{x}\left(x, y_{n_{0}}\right)\right) \psi_{n}\right|^{2} d x \\
& \geq s \int_{I_{0}}\left|j_{x}^{\left(n_{0}\right)}(x)\right|^{2} d x \geq|\ln s|^{2},
\end{aligned}
$$

a contradiction for $s$ sufficiently small.

Now from the equation of conservation of current (13) and the definition of $j_{y}^{(n)}$ (see (11)), we conclude that

$$
\left|\partial_{x} j_{x}^{(n)}\right| \leq \frac{2}{s^{2}}
$$

For any $n, x \in[-L, L]$ we know from the preceding paragraph that there exists $x_{0}$ with $\left|x-x_{0}\right| \leq s$ and $\left|j_{x}^{(n)}\left(x_{0}\right)\right| \leq \frac{1}{s}|\ln s|$. Clearly we then have

$$
\left|j_{x}^{(n)}(x)\right| \leq\left|j_{x}^{(n)}\left(x_{0}\right)\right|+\left|\int_{x_{0}}^{x} \partial_{x} j_{x}^{(n)}(x) d x\right| \leq \frac{3}{s}|\ln s| .
$$


Finally, we turn to the field $h(x, y)=h^{(n)}(x), y_{n-1}<y<y_{n}$. By the same argument as above, the bound on energy implies that for every $n$ and for every interval $I$ of width at least $s$ there must exist $x_{0} \in I$ with $h^{(n)}\left(x_{0}\right) \leq \frac{1}{s}|\ln s|$. From the Euler-Lagrange equation (11) we have $\left|\partial_{x} h^{(n)}(x)\right| \leq 1 / s$, and so we obtain the estimate (22) in the same way as (21).

\section{LOWER BOUNDS ON THE ENERGY}

In this section we prove a lower bound which matches the upper bound of Proposition 3.1. Using a technique due to Sandier [S] (see also Jerrard [Je] for a similar result) we show that the minimizing solutions of the Lawrence-Doniach model in the limit described by (H1), (H2) have a vortex structure.

4.1. Extending $\psi_{n}$ to $\mathbf{R}^{2}$. For $\left(\psi_{n}, \vec{A}\right) \in t \mathcal{H}_{K}^{*}$ we define the following extension of $\psi_{n}$ to all of $\mathbf{R}^{2}$ : for $y_{n-1}<y \leq y_{n}$ let

$$
\Psi(x, y):=\left[\frac{y-y_{n-1}}{s} \psi_{n}(x)+\frac{y_{n}-y}{s} \psi_{n-1}(x) e^{i \int_{y_{n-1}}^{y_{n}} A_{y}(x, y) d y}\right] e^{-i \int_{y}^{y_{n}} A_{y}(x, u) d u} .
$$

Then $\Psi \in H^{1}(\Omega), \Psi\left(x, y_{n}\right)=\psi_{n}(x)$ for all $n, x$. Moreover, if $\omega_{x}, \omega_{y}$ are the functions associated to $\left(\psi_{n}, \vec{A}\right) \in t \mathcal{H}_{K}^{*}$, then $\Psi$ satisfies the 't Hooft conditions

$$
\Psi(x+2 L, y)=\Psi(x, y) e^{i \omega_{x}}, \quad \Psi(x, y+2 M)=\Psi(x, y) e^{i \omega_{y}}
$$

with the same $\omega_{x}, \omega_{y}$. In particular (see [Bo] for instance) we have

Proposition 4.1. Let $T$ be the flat torus with fundamental domain $\Omega$. Let $\mathbb{L}$ be the complex line bundle with Euler number $K=\operatorname{deg}(\Psi, 0)$ over $T$. For any $\left(\psi_{n}, \vec{A}\right) \in t \mathcal{H}_{K}^{*}$, the function $\Psi$ defined by (23) defines a section of $\mathbb{L}$ and $\vec{A}$ a connection 1 -form on $\mathbb{L}$.

We now connect the terms of the Lawrence-Doniach energy to derivatives of $\Psi$. For convenience we define:

$$
t=t(y)=\frac{y-y_{n-1}}{s} \quad \text { for } y_{n-1}<y \leq y_{n} .
$$

Note that $t(y)$ is $s$-periodic in $y$. We also denote $D_{x}^{(n)}:=\partial_{x}-i A_{x}\left(\cdot, y_{n}\right)$.

Lemma 4.2. Assume $\left(\psi_{n}, \vec{A}\right) \in t \mathcal{H}_{K}^{*}$ are critical points of $\mathcal{E}_{\kappa, s}^{L D}$ with $\mathcal{E}_{\kappa, s}^{L D}\left(\psi_{n}, \vec{A}\right) \leq$ $C_{0}|\ln s|$. Then for $y \in\left(y_{n-1}, y_{n}\right)$,

$$
\left|\left(\partial_{y}-i A_{y}(x, y)\right) \Psi(x, y)\right|^{2}=\frac{1}{s^{2}}\left|\psi_{n}-\psi_{n-1} e^{i \int_{y_{n-1}}^{y_{n}} A_{y}(x, y) d y}\right|^{2},
$$

$$
\begin{aligned}
\left|\left(\partial_{x}-i A_{x}(x, y)\right) \Psi(x, y)\right|^{2} \leq & t(y)\left|D_{x}^{(n)} \psi_{n}(x)\right|^{2}+(1-t(y))\left|D_{x}^{(n-1)} \psi_{n-1}\right|^{2}+g_{n}(x, y), \\
(27) \quad\left(|\Psi(x, y)|^{2}-1\right)^{2} \leq & 2\left[t\left(\left|\psi_{n}\right|^{2}-1\right)^{2}+(1-t)\left(\left|\psi_{n-1}\right|^{2}-1\right)^{2}\right] \\
& +\frac{1}{2}\left|\psi_{n}-\psi_{n-1} e^{i \int_{y_{n-1}}^{y_{n}} A_{y}(x, y) d y}\right|^{2}
\end{aligned}
$$

where $g_{n}(x, y) \geq 0$ and

$$
0 \leq \sum_{n=1-N}^{N} \int_{-L}^{L} \int_{y_{n-1}}^{y_{n}} g_{n}(x, y) d x d y \rightarrow 0 \quad \text { as } s \rightarrow 0 .
$$


Remark 4.3. An immediate consequence of Lemma 4.2 are the following inequalities which suggest a connection between the Lawrence-Doniach energy for $\left(\psi_{n}, \vec{A}\right)$ and the Ginzburg-Landau energy of $(\Psi, \vec{A})$ :

$$
\begin{aligned}
& \iint_{\Omega}|(\nabla-i \vec{A}) \Psi(x, y)|^{2} d x d y \\
& \leq s \sum_{n=1-N}^{N} \int_{-L}^{L}\left\{\left|\left(\partial_{x}-i A_{x}\left(x, y_{n}\right)\right) \psi_{n}\right|^{2} .\right. \\
& \left.\quad+\frac{1}{s^{2}}\left|\psi_{n}-\psi_{n-1} e^{i \int_{y_{n-1}}^{y_{n}} A_{y}(x, y) d y}\right|^{2}\right\} d x+o(1)
\end{aligned}
$$

$$
\begin{aligned}
& \iint_{\Omega} \frac{1}{s^{2}}\left(1-|\Psi(x, y)|^{2}\right)^{2} d x d y \\
& \quad \leq s \sum_{n=1-N}^{N}\left[\frac{2}{s^{2}} \int_{-L}^{L}\left(1-\left|\psi_{n}\right|^{2}\right)^{2} d x+\frac{1}{2 s^{2}} \int_{-L}^{L}\left|\psi_{n}-\psi_{n-1} e^{i \int_{y_{n-1}}^{y_{n}} A_{y}(x, y) d y}\right|^{2} d x\right],
\end{aligned}
$$

where $o(1)$ denotes a quantity which tends to zero as $s \rightarrow 0$. Note that $\mathcal{E}_{\kappa, s}^{L D}\left(\psi_{n}, \vec{A}\right)$ does not exactly control the Ginzburg-Landau energy of $(\Psi, \vec{A})$, since the Josephsoncoupling term in $\mathcal{E}_{\kappa, s}^{L D}$ is needed in both (29) and (30). This is because $|\Psi|$ could be small or vanish (in the gap between superconducting planes) even when $\left|\psi_{n}\right|$ remains near 1 . Indeed, this is exactly what happens at a vortex: the contribution to the energy comes from the turning of the complex phase, which is measured by the Josephson-coupling term.

Proof. We calculate for $y_{n-1}<y \leq y_{n}$,

$$
\begin{aligned}
\left(\partial_{y}-i A_{y}(x, y)\right) \Psi(x, y)= & \frac{1}{s}\left(\psi_{n}(x)-\psi_{n-1} e^{i \int_{y_{n-1}}^{y_{n}} A_{y}(x, y) d y}\right) \\
\left(\partial_{x}-i A_{x}(x, y)\right) \Psi(x, y)= & e^{-i \int_{y}^{y_{n}} A_{y} d y}\left\{t\left[\left(\partial_{x}-i A_{x}\left(x, y_{n}\right)\right) \psi_{n}\right]\right. \\
& +(1-t)\left[\left(\partial_{x}-i A_{x}\left(x, y_{n-1}\right)\right) \psi_{n-1} e^{i \int_{y_{n-1}}^{y_{n}} A_{y}(x, y) d y}\right. \\
& \left.-i h^{(n)}(x) \operatorname{st}(1-t)\left[\psi_{n}-\psi_{n-1} e^{i \int_{y_{n-1}}^{y_{n}} A_{y}(x, y) d y}\right]\right\}
\end{aligned}
$$

The identity (31) is already in the desired form. We use (32) to estimate the covariant $x$-derivative of $\Psi$ : we let $\epsilon=1 /|\ln s|^{2}$ and calculate for any $y \in\left(y_{n-1}, y_{n}\right)$,

$$
\begin{aligned}
\left|\left(\partial_{x}-i A_{x}(x, y)\right) \Psi\right|^{2} \leq & (1+\epsilon)\left|t D_{x}^{(n)} \psi_{n}+(1-t) D_{x}^{(n-1)} \psi_{n-1} e^{i \int_{y_{n-1}}^{y_{n}} A_{y}(x, y) d y}\right|^{2} \\
& +\frac{2}{\epsilon} \sup _{x, n}\left|h^{(n)}(x) s\right|^{2} \cdot \frac{1}{16}\left|\psi_{n}-\psi_{n-1} e^{i \int_{y_{n-1}}^{y_{n}} A_{y}(x, y) d y}\right|^{2} \\
\leq & (1+\epsilon)\left[t\left|D_{x}^{(n)} \psi_{n}\right|^{2}+(1-t)\left|D_{x}^{(n-1)} \psi_{n-1}\right|^{2}\right] \\
& +\frac{1}{8 \epsilon} \sup _{x, n}\left|h^{(n)}(x) s\right|^{2} \cdot\left|\psi_{n}-\psi_{n-1} e^{i \int_{y_{n-1}}^{y_{n}} A_{y}(x, y) d y}\right|^{2}
\end{aligned}
$$

by convexity. 
Let

$$
\begin{aligned}
g_{n}(x, y):=\epsilon\left[t\left|D_{x}^{(n)} \psi_{n}\right|^{2}+(1-t)\left|D_{x}^{(n-1)} \psi_{n-1}\right|^{2}\right] \\
\quad+\frac{1}{8 \epsilon} \sup _{x, n}\left|h^{(n)}(x) s\right|^{2} \cdot\left|\psi_{n}-\psi_{n-1} e^{i \int_{y_{n-1}}^{y_{n}} A_{y}(x, y) d y}\right|^{2} .
\end{aligned}
$$

To verify (26) it suffices to show (28). The second term above can be estimated using Lemma 3.2:

$$
\begin{aligned}
& \sum_{n=1-N}^{N} \frac{1}{8 \epsilon} \sup _{x, n}\left|h^{(n)}(x) s\right|^{2} \int_{y_{n-1}}^{y_{n}} \int_{-L}^{L}\left|\psi_{n}-\psi_{n-1} e^{i \int_{y_{n-1}}^{y_{n}} A_{y}(x, y) d y}\right|^{2} d x d y \\
& \quad \leq \frac{1}{8 \epsilon} \cdot 4|\ln s|^{2} \cdot s \sum_{n=1-N}^{N} \int_{-L}^{L}\left|\psi_{n}-\psi_{n-1} e^{i \int_{y_{n-1}}^{y_{n}} A_{y}(x, y) d y}\right|^{2} d x \\
& \leq \frac{s^{2}|\ln s|^{2}}{2 \epsilon} \mathcal{E}_{\kappa, s}^{L D}\left(\psi_{n}, \vec{A}\right) \leq C s^{2}|\ln s|^{5}=o(1) .
\end{aligned}
$$

By the periodicity (in $n$ ) of $D_{x}^{(n)} \psi_{n}$ and $t=t(y)$ we then have

$$
\begin{aligned}
& \sum_{n=1-N}^{N} \int_{y_{n-1}}^{y_{n}} \int_{-L}^{L} g_{n}(x, y) d x d y \\
& \quad \leq \epsilon \sum_{n=1-N}^{N} \int_{y_{n-1}}^{y_{n}} \int_{-L}^{L}\left\{t\left|D_{x}^{(n)} \psi_{n}\right|^{2}+(1-t)\left|D_{x}^{(n-1)} \psi_{n-1}\right|^{2}\right\} d x d y+o(1) \\
& \quad=\epsilon \sum_{n=1-N}^{N} \int_{y_{n-1}}^{y_{n}} \int_{-L}^{L}\left|D_{x}^{(n)} \psi_{n}\right|^{2} d x d y \leq \epsilon \mathcal{E}_{\kappa, s}^{L D}\left(\psi_{n}, \vec{A}\right)+o(1)=o(1),
\end{aligned}
$$

using the upperbound on energy, (15). This verifies (26) and (28).

Next we verify $(27)$. For any $y \in\left(y_{n-1}, y_{n}\right)$, we have:

$$
\begin{aligned}
&\left(|\Psi|^{2}-1\right)^{2} \\
&=\left(2 t(1-t) \operatorname{Re}\left\{\psi_{n}^{*} \psi_{n-1} e^{i \int_{y_{n-1}}^{y_{n}} A_{y}(x, y) d y}\right\}+t^{2}\left|\psi_{n}\right|^{2}+(1-t)^{2}\left|\psi_{n-1}\right|^{2}-1\right)^{2} \\
&=\left(-t(1-t)\left|\psi_{n}-\psi_{n-1} e^{i \int_{y_{n-1}}^{y_{n}} A_{y}(x, y) d y}\right|^{2}+t\left(\left|\psi_{n}\right|^{2}-1\right)\right. \\
&\left.\quad+(1-t)\left(\left|\psi_{n-1}\right|^{2}-1\right)\right)^{2} \\
& \leq 2\left[t\left(\left|\psi_{n}\right|^{2}-1\right)+(1-t)\left(\left|\psi_{n-1}\right|^{2}-1\right)\right]^{2} \\
& \quad+2 t^{2}(1-t)^{2}\left|\psi_{n}-\psi_{n-1} e^{i \int_{y_{n-1}}^{y_{n}} A_{y}(x, y) d y}\right|^{4} \\
& \leq 2\left[t\left(\left|\psi_{n}\right|^{2}-1\right)^{2}+(1-t)\left(\left|\psi_{n-1}\right|^{2}-1\right)^{2}\right] \\
& \quad+\frac{1}{2}\left|\psi_{n}-\psi_{n-1} e^{i \int_{y_{n}-1}^{y_{n}} A_{y}(x, y) d y}\right|^{2}
\end{aligned}
$$

where in the last line we use Jensen's inequality and the bound $\left|\psi_{n}\right| \leq 1$. This concludes the proof of Lemma 4.2. 
4.2. A comparison functional. We now introduce a comparison functional of Ginzburg-Landau type which we use to establish a tight lower bound for $\mathcal{E}_{\kappa, s}^{L D}$ that will allow us to describe the vortex structure of minimizers, in the spirit of [S], [Je].

Let $\gamma>0$ be as in (H2) and define

$$
\alpha:=\min \left\{\gamma^{2}, 1\right\} .
$$

We define the following auxilliary functional: for $\omega \subset \Omega$ :

$$
\begin{array}{r}
\mathcal{M}_{s, \omega}(\Psi, \vec{A}):=\iint_{\omega}\left\{|\Psi|^{2}|(\nabla-i \vec{A}) \Psi|^{2}+\frac{1}{2}\left(1-|\Psi|^{2}\right)\left[\left.|\nabla| \Psi\right|^{2}+\frac{\alpha}{s^{2}}\left(1-|\Psi|^{2}\right)^{2}\right]\right. \\
\left.+(\operatorname{curl} \vec{A})^{2}\right\} d x d y
\end{array}
$$

Lemma 4.4. Under the hypotheses of Lemma 4.2,

$$
\mathcal{E}_{\kappa, s}^{L D}\left(\psi_{n}, \vec{A}\right) \geq \mathcal{M}_{s, \Omega}(\Psi, \vec{A})+o(1) .
$$

Proof. First, from Lemma 4.2 we have for every $y \in\left(y_{n-1}, y_{n}\right)$,

$$
\begin{aligned}
\frac{1}{2}|\nabla(|\Psi|)|^{2} & +\frac{\alpha}{2 s^{2}}\left(1-|\Psi|^{2}\right)^{2} \\
\leq & \frac{1}{2}|(\nabla-i \vec{A}) \Psi|^{2}+\frac{\alpha}{2 s^{2}}\left(1-|\Psi|^{2}\right)^{2} \\
\leq & \frac{1}{2}\left\{t\left|D_{x}^{(n)} \psi_{n}\right|^{2}+(1-t)\left|D_{x}^{(n-1)} \psi_{n-1}\right|^{2}\right\}+\frac{1}{s^{2}}\left|\psi_{n}-\psi_{n-1} e^{i \int_{y_{n-1}}^{y_{n}} A_{y}(x, y) d y}\right|^{2} \\
& \quad+\kappa^{2}\left\{t\left(1-\left|\psi_{n}\right|^{2}\right)^{2}+(1-t)\left(1-\left|\psi_{n-1}\right|^{2}\right)^{2}\right\}+\frac{1}{2} g_{n}(x, y) .
\end{aligned}
$$

Hence,

$$
\begin{aligned}
&\left\{t\left|D_{x}^{(n)} \psi_{n}\right|^{2}+(1-t)\left|D_{x}^{(n-1)} \psi_{n-1}\right|^{2}\right\}+\frac{1}{s^{2}}\left|\psi_{n}-\psi_{n-1} e^{i \int_{y_{n-1}}^{y_{n}} A_{y}(x, y) d y}\right|^{2} \\
&+\kappa^{2}\left\{t\left(1-\left|\psi_{n}\right|^{2}\right)^{2}+(1-t)\left(1-\left|\psi_{n-1}\right|^{2}\right)^{2}\right\}+g_{n}(x, y) \\
& \geq \max \left\{|(\nabla-i \vec{A}) \Psi|^{2}, \frac{1}{2}|\nabla(|\Psi|)|^{2}+\frac{\alpha}{2 s^{2}}\left(1-|\Psi|^{2}\right)^{2}\right\} \\
& \geq|\Psi|^{2}|(\nabla-i \vec{A}) \Psi|^{2}+\left(1-|\Psi|^{2}\right)\left[\frac{1}{2}|\nabla(|\Psi|)|^{2}+\frac{\alpha}{2 s^{2}}\left(1-|\Psi|^{2}\right)^{2}\right],
\end{aligned}
$$

since $0 \leq|\Psi(x, y)|^{2} \leq 1$ and $\max \{A, B\} \geq \lambda A+(1-\lambda) B$ for $\lambda \in[0,1]$. Integrating over strips and summing over $n$ we arrive at (34).

Remark 4.5. Note that the lower bound (34) remains valid if we restrict to a smooth subdomain of $\Omega$, since the inequality is local.

4.3. Lower bound for the comparison functional. We bound from below the functional $\mathcal{M}$ using the strategy of $[\mathrm{S}]$. We first state a result of $[\mathrm{S}]$ adapted to our setting.

4.3.1. A lower bound on the perforated torus.

Definition 4.6. The radius of a compact set $\omega \subset T$ is the infimum over all finite coverings of $\omega$ by open balls $B_{1}, \ldots, B_{k}$ of the sum $r_{1}+\cdots+r_{k}$, where $r_{i}$ is the radius of $B_{i}$. We write $r(\omega)$ for this quantity. 
Note that in this definition we may assume the coverings are disjoint. Indeed if $B_{1}=B\left(a_{1}, r_{1}\right)$ and $B_{2}=B\left(a_{2}, r_{2}\right)$ are intersecting balls in $T$ - or even if $\bar{B}_{1} \cap \bar{B}_{2} \neq \varnothing$, then

$$
B_{1} \cup B_{2} \subset B\left(\frac{r_{1} a_{1}+r_{2} a_{2}}{r_{1}+r_{2}}, r_{1}+r_{2}\right) .
$$

Using this to group together intersecting balls, a finite covering may be replaced by a disjoint covering - or even a covering by balls with disjoint closures - leaving the sum of radii unchanged. It is also clear that $r(A \cup B) \leq r(A)+r(B)$.

There is a relationship between radius and perimeter:

Lemma 4.7. Assume $\omega$ is a compact subset of $T$ and $\mathcal{H}^{1}(\partial \omega)<\min (M, L)$. Then either $r(\omega) \leq \mathcal{H}^{1}(\partial \omega)$ or $r(T \backslash \omega) \leq \mathcal{H}^{1}(\partial \omega)$.

(Here, $\mathcal{H}^{1}(\cdot)$ denotes 1-dimensional Haudorff measure.)

Proof. It suffices to show that if $\left\{B_{i}\right\}_{i \in \mathbb{N}}$ is any covering of $\partial \omega$ by open balls of radii $\left\{r_{i}\right\}_{i \in \mathbb{N}}$ such that $\sum_{i} r_{i}<\min (M, L)$, then $\omega \subset \sum_{i} r_{i}$ or $T \backslash \omega \subset \sum_{i} r_{i}$. Since $\partial \omega$ is compact we may extract a finite covering, and then using the remark above we may assume the balls are disjoint. Now if $B_{1}, \ldots, B_{k}$ are disjoint open balls covering $\partial \omega$ and the sum of their radii is smaller than $\min (M, L)$, we claim they cover $\omega$ or $T \backslash \omega$, which concludes the proof.

The claim follows by noting that since $\sum_{i} r_{i}<\min (M, L)$ the set $A=T \backslash \bigcup_{i=1}^{k} B_{i}$ is connected and thus if $A$ intersected both $\omega$ and its complement it would also intersect $\partial \omega$, which is impossible from the definition of $A$. Therefore either $T \backslash \omega \subset$ $\bigcup_{i=1}^{k} B_{i}$ or $\omega \subset \bigcup_{i=1}^{k} B_{i}$.

We will use the following variant of a result in [S] (see [Je] for a similar construction)

Proposition 4.8. Let $\omega$ be a compact subset of the flat two dimensional torus $T$. Then for any $\min (L, M, 1)>\eta>\alpha>r(\omega)$ there exists a collection $\left\{B_{i}\right\}_{1 \leq i \leq k}$ of disjoint open balls with radii $\left\{r_{i}\right\}_{1 \leq i \leq k}$ such that 1) $r_{1}+\cdots+r_{k} \leq \eta$, 2) $\omega \subset \bigcup_{i} B_{i}$, 3) for any section $u$ of $\mathbb{L}$ such that $|u| \equiv 1$ over $T \backslash \omega$, any connection $A$ on $\mathbb{L}$ and any $1 \leq i \leq k$,

$$
\iint_{B_{i} \backslash \omega}|(\nabla-i \vec{A}) u|^{2}+\iint_{B_{i}}(\operatorname{curl} \vec{A})^{2} d x d y \geq 2 \pi\left|d_{i}\right|\left(\log \frac{\eta}{\alpha}-\frac{1}{2}\right),
$$

where $d_{i}=\operatorname{deg}\left(u, \partial B_{i}\right)$.

In $[S]$ a version of this is proved in the setting of a domain in $\mathbb{R}^{2}$ with boundary. The proof is actually simpler in our case from the absence of boundary; we omit it.

4.3.2. Application of the coarea formula. We begin with some notations.

Definition 4.9. Let $\Psi, \vec{A}$ be respectively a section of $\mathbb{L}$ and a connection on $\mathbb{L}$ where $\mathbb{L}$ is a complex line bundle over $T$. We define $\rho=|\Psi|, u=\Psi /|\Psi|$ and for $t>0$

$$
\omega_{t}=\{|\Psi| \leq t\}, \quad \Omega_{t}=\{|\Psi|>t\}, \quad \Theta(t)=\iint_{\Omega_{t}}|(\nabla-i \vec{A}) u|^{2}+\iint_{\Omega}(\operatorname{curl} \vec{A})^{2} .
$$


Also, we let

$$
\begin{aligned}
\mathcal{T} & =\iint_{T} \rho^{4}|(\nabla-i \vec{A}) u|^{2}+(\operatorname{curl} \vec{A})^{2} \\
\mathcal{N} & =\iint_{T} \rho^{2}|\nabla \rho|^{2}+\frac{1}{2}\left(1-\rho^{2}\right)\left(|\nabla \rho|^{2}+\frac{\alpha}{s^{2}}\left(1-\rho^{2}\right)^{2}\right) .
\end{aligned}
$$

We have

Lemma 4.10. Using the above notations $\mathcal{M}_{s, T}(\Psi, \vec{A})=\mathcal{T}+\mathcal{N}$ and

$$
\mathcal{T} \geq \int_{0}^{1} 4 t^{3} \Theta(t) d t, \quad \mathcal{N} \geq \int_{t=0}^{1} \frac{f(t) \mathcal{H}^{1}(\{\rho=t\})}{s} d t
$$

where

$$
f(t)=\sqrt{2} \alpha^{\frac{1}{2}} t\left(1-t^{2}\right)^{\frac{3}{2}} .
$$

Proof. The fact that $\mathcal{M}_{s, \Omega}(\Psi, \vec{A})=\mathcal{T}+\mathcal{N}$ follows directly from definitions (33),(38) using the fact that

$$
|(\nabla-i \vec{A}) \Psi|^{2}=\rho^{2}|(\nabla-i \vec{A}) u|^{2}+|\nabla \rho|^{2} .
$$

On the other hand,

$$
\begin{gathered}
\iint_{\Omega} \rho^{2}|\nabla \rho|^{2}+\frac{1}{2}\left(1-\rho^{2}\right)\left[|\nabla \rho|^{2}+\frac{\alpha}{s^{2}}\left(1-|\rho|^{2}\right)^{2}\right] d x d y \\
\geq \iint_{\Omega} \rho^{2}|\nabla \rho|^{2}+\frac{\alpha}{2 s^{2}}\left(1-\rho^{2}\right)^{3} d x d y
\end{gathered}
$$

therefore

$$
\mathcal{N} \geq \iint_{\Omega} \rho^{2}|\nabla \rho|^{2}+\frac{\alpha}{2 s^{2}}\left(1-\rho^{2}\right)^{3} d x d y
$$

The coarea formula yields

$$
\iint_{\Omega} \rho^{2}|\nabla \rho|^{2}+\frac{\alpha}{2 s^{2}}\left(1-\rho^{2}\right)^{3} d x d y=\int_{0}^{+\infty}\left[\int_{\{\rho=t\}} t^{2}|\nabla \rho|+\frac{\alpha}{2 s^{2}} \frac{\left(1-t^{2}\right)^{3}}{|\nabla \rho|} d \mathcal{H}^{1}\right] d t
$$

Using Cauchy-Schwarz's inequality

$$
\begin{aligned}
\int_{0}^{+\infty} & {\left[\int_{\{\rho=t\}} t^{2}|\nabla \rho|+\frac{\alpha}{2 s^{2}} \frac{\left(1-t^{2}\right)^{3}}{|\nabla \rho|} d \mathcal{H}^{1}\right] d t } \\
\geq & \int_{0}^{+\infty} \frac{\sqrt{2} \alpha^{\frac{1}{2}} t\left(1-t^{2}\right)^{\frac{3}{2}}}{s} \mathcal{H}^{1}(\{\rho=t\}) d t,
\end{aligned}
$$

which proves the lower bound for $\mathcal{N}$. On the other hand, Fubini's Theorem implies

$$
\begin{aligned}
\iint_{\Omega} \rho^{4}|(\nabla-i \vec{A}) u|^{2} d x d y & =-\int_{0}^{+\infty} t^{4} \frac{d}{d t}\left(\int_{\Omega_{t}}|(\nabla-i \vec{A}) u|^{2}\right) d t \\
& =\int_{0}^{+\infty} 4 t^{3}\left(\int_{\Omega_{t}}|(\nabla-i \vec{A}) u|^{2}\right) d t
\end{aligned}
$$

while

$$
\iint_{\Omega}(\operatorname{curl} \vec{A})^{2} d x d y=\int_{0}^{1} 4 t^{3}\left(\iint_{\Omega}(\operatorname{curl} \vec{A})^{2}\right) d t
$$


Therefore

$$
\mathcal{T} \geq \int_{t=0}^{1} 4 t^{3} \Theta(t) d t
$$

The lemma is proved.

4.3.3. Lower bound. We first prove

Proposition 4.11. For any $C_{0}>0$ there exist $s_{0}, C>0$ such that if $\Psi, \vec{A}$ are respectively a section of $\mathbb{L}$ and a connection on $\mathbb{L}$ where $\mathbb{L}$ is a complex line bundle over $T$ of degree $K$ satisfying $\mathcal{M}_{s, \Omega}(\Psi, \vec{A}) \leq C_{0}|\ln s|$ for some $s<s_{0}$, the following holds for almost every $0<t<1-C \sqrt{s}$ :

$$
4 t^{3} \Theta(t) \geq 8 \pi K t^{3} \log \frac{1}{x(t)}+g(t),
$$

where $\Theta(t)$ is defined in (37) and $x(t)=f(t) \mathcal{H}^{1}(\{\rho=t\})$, using the notations in Definition 4.9 and (40). Moreover

$$
\|g\|_{L^{1}(0,1-C \sqrt{s})} \leq C .
$$

Proof. From Proposition 4.8, applied with $\eta=\min (L, M, 1)$ and $\omega=\omega_{t}=\{\rho \leq t\}$, we find, letting $\gamma_{t}=\{\rho=t\}$,

$$
\Theta(t) \geq 2 \pi K\left(\log \frac{1}{r\left(\omega_{t}\right)}-C\right) \geq 2 \pi K\left(\log \frac{1}{x(t)}+\log (f(t))-\log _{+} \frac{r\left(\omega_{t}\right)}{\mathcal{H}^{1}\left(\gamma_{t}\right)}-C\right),
$$

where $C$ is a constant independent of $\Psi, \vec{A}, s$ and $\log _{+}=\max (\log , 0)$. Note that the coarea formula assures that for almost every $t, \gamma_{t}=\partial \omega_{t}$ has finite one-dimensional Hausdorff measure.

Since $\log f(t)$ is integrable on $[0,1]$, it remains to prove that $\log _{+}\left(r\left(\omega_{t}\right) / \mathcal{H}^{1}\left(\gamma_{t}\right)\right)$ has $L^{1}$ norm bounded independent of $s, \Psi, \vec{A}$ on $(0,1-C \sqrt{s})$ for $s$ small enough and $C$ large enough. From Lemma 4.7, for given $0 \leq t \leq 1$ three cases might occur:

1) $\mathcal{H}^{1}\left(\gamma_{t}\right) \geq \min (M, L)$.

2) $r\left(\omega_{t}\right) \leq \mathcal{H}^{1}\left(\gamma_{t}\right) \leq \min (M, L)$.

3) $r\left(\Omega_{t}\right) \leq \mathcal{H}^{1}\left(\gamma_{t}\right) \leq \min (M, L)$.

Case 3) cannot occur for small $t$. Indeed if $r\left(\Omega_{t}\right) \leq \mathcal{H}^{1}\left(\gamma_{t}\right) \leq \min (M, L)$, then the isoperimetric inequality implies that $\left|\Omega_{t}\right| \leq|T| / 2$, where $|$.$| denotes the area.$ Thus in this case $\left|\omega_{t}\right| \geq C$, where $C$ is a positive constant and therefore

Thus

$$
C_{0}|\ln s| \geq \mathcal{M}_{s, \Omega}(\Psi, \vec{A}) \geq \frac{C \alpha}{2 s^{2}}\left(1-t^{2}\right)^{3} .
$$

$$
1-t \leq C\left(\frac{s^{2} \ln s}{\alpha}\right)^{\frac{1}{3}} \leq C s^{\frac{1}{2}}
$$

if $s$ is small enough. Thus if $s$ is small enough, $C$ large enough and $0<t<1-C \sqrt{s}$, then case 3) cannot occur. But in either case 1) or 2) it is clear that $r\left(\omega_{t}\right) / \mathcal{H}^{1}\left(\gamma_{t}\right)$ is bounded from above by a constant, hence

$$
\left\|\log _{+}\left(r\left(\omega_{t}\right) / \mathcal{H}^{1}\left(\gamma_{t}\right)\right)\right\|_{L^{1}(0,1-C \sqrt{s})} \leq C .
$$

The proposition is proved.

As a consequence of the above we deduce the following. 
Proposition 4.12. For any $C_{0}>0$ there exist $s_{0}, C>0$ such that if $\left(\psi_{n}, \vec{A}\right) \in t \mathcal{H}_{K}^{*}$ satisfies $\mathcal{E}_{\kappa, s}^{L D}\left(\psi_{n}, \vec{A}\right) \leq C_{0}|\ln s|$ with $s<s_{0}$, then

$$
\mathcal{M}_{s, \Omega}(\Psi, \vec{A}) \geq \int_{0}^{1-C \sqrt{s}} \frac{x(t)}{s}+4 t^{3} \Theta(t) d t \geq 2 \pi K\left(\log \frac{1}{s}-C\right),
$$

where $\Psi(x, y)$ is defined as in (23).

Proof. From Lemma 4.4 we have for $s$ small enough $\mathcal{M}_{s, \Omega}(\Psi, \vec{A}) \leq 2 C_{0}|\ln s|$ and therefore Proposition 4.11 applies and yields, using Lemma 4.10,

$$
\mathcal{M}_{s, \Omega}(\Psi, \vec{A}) \geq \int_{0}^{1-C \sqrt{s}} \frac{x}{s}+8 \pi K t^{3} \log \frac{1}{x} d t-C .
$$

Minimizing for each $t$ the integrand above with respect to $x$,

$$
\frac{x}{s}+8 \pi K t^{3} \log \frac{1}{x} \geq 8 \pi K t^{3} \log \frac{1}{s}+h(t),
$$

where $h$ is a function with $L^{1}$ norm bounded independent of $s$ in $[0,1]$. This proves the proposition.

4.4. Energy concentration. The lower bound computed above, together with a matching upper bound, allows us to prove the concentration of energy in small balls (see [S], [Je]). More precisely, let $\left(\psi_{n, s}, \vec{A}_{s}\right)$ be a minimizer of $\mathcal{E}_{\kappa, s}^{L D}$ with parameter $s$. Define $\Psi_{s}$ by (23). Then

Proposition 4.13. For every $\sigma>0$ there exists a subsequence $s_{k} \rightarrow 0$, a finite collection of points $\left\{a_{1}, \ldots, a_{m}\right\} \subset \Omega$ with $m \leq K$ and a constant $M_{0}=M_{0}(\sigma)$ such that

$$
\iint_{T \backslash \bigcup_{i} B\left(a_{i}, \sigma\right)}\left|\left(\nabla-i \vec{A}_{s_{k}}\right) \Psi_{s_{k}}\right|^{2}+\left(\operatorname{curl} \vec{A}_{s_{k}}\right)^{2} d x d y \leq M_{0}
$$

uniformly in $s=s_{k}$.

Before proving Proposition 4.13 we begin by listing direct consequences of matching upper and lower bounds.

Lemma 4.14. For any $C_{0}>0$ there exist $s_{0}, C>0$ such that if $\Psi, \vec{A}$ are respectively a section of $\mathbb{L}$ and a connection on $\mathbb{L}$ where $\mathbb{L}$ is a complex line bundle over $T$ of degree $K$ satisfying

$$
2 \pi K|\ln s|-C_{0} \leq \mathcal{M}_{s, \Omega}(\Psi, \vec{A}) \leq 2 \pi K|\ln s|+C_{0}
$$

for some $s<s_{0}$, then

$$
\begin{gathered}
\mathcal{N}-\int_{0}^{1-C \sqrt{s}} \frac{x(t)}{s} d t \leq C, \quad \mathcal{T}-\int_{0}^{1-C \sqrt{s}} 4 t^{3} \Theta(t) d t \leq C, \\
\int_{0}^{1-C \sqrt{s}}\left|4 \Theta(t)-8 \pi K \log \frac{1}{x(t)}\right| t^{3} d t \leq C, \\
\int_{0}^{1-C \sqrt{s}}\left|\frac{x(t)}{s}+8 \pi K t^{3} \log \frac{s}{x(t)}\right| d t \leq C, \\
\int_{1-C \sqrt{s}}^{1} 4 t^{3} \Theta(t) d t \leq C,
\end{gathered}
$$

where we have used the notations of Definition 4.9 and Proposition 4.11. 
Proof. Each inequality is a consequence of two bounds: first, the integrals of each quantity have upper and lower bounds which match up to a constant; this follows from Lemma 4.10, Proposition 4.11 and Proposition 4.12. Second, each integrand is pointwise bounded below by an $L^{1}$ function. For example, to show the second inequality of (50) we note that Proposition 4.12 and the upper bound on the energy of minimizers ensures that

$$
C_{1} \leq \int_{0}^{1-C \sqrt{s}} \frac{x}{s}+8 \pi K t^{3} \log \frac{s}{x} d t \leq C_{2} .
$$

Minimizing the integrand pointwise with respect to $x$ (for $t$ fixed) gives the same pointwise lower bound (48), with $\|h\|_{L^{1}}$ uniformly bounded. Then, $\frac{x}{s}+8 \pi K t^{3} \log \frac{s}{x}$ $-h(t) \geq 0$ and hence has bounded $L^{1}$-norm, so by the reverse triangle inequality we arrive at the desired inequality. The other estimates are proved in a similar way.

We will also need

Lemma 4.15. Under the assumptions of the previous lemma, and using the same notation,

$$
\mathcal{N} \leq C, \quad \int_{0}^{1}\left|4 \Theta(t)-8 \pi K \log \frac{1}{s}\right| t^{3} d t \leq C .
$$

Proof. We first prove that $\mathcal{N} \leq C$. In view of (49), it suffices to proves that $x(t) / s$ is bounded in $L^{1}(0,1-C \sqrt{s})$ independent of $s$. But where $x(t)>s$, the inequality

$$
\log \frac{x}{s} \leq C \sqrt{\frac{x}{s}}
$$

holds for $C$ large enough, hence from (50) we find that the $L^{1}$ norm of $x / s-C \sqrt{x / s}$ on $(0,1-C \sqrt{s})$ is bounded independent of $s$. Thus the same is true for $x / s$, and from (49), we get $\mathcal{N} \leq C$.

Using the above together with (50) we get

$$
\int_{0}^{1-C \sqrt{s}}\left|8 \pi K t^{3} \log \frac{s}{x(t)}\right| d t \leq C
$$

and then

$$
\int_{0}^{1-C \sqrt{s}}\left|4 t^{3} \Theta(t)-8 \pi K t^{3} \log \frac{1}{s}\right| d t \leq C .
$$

From (51) and the fact that the integral of $8 \pi K t^{3} \log (1 / s)$ on $(1-C \sqrt{s}, 1)$ is bounded independent of $s$, we conclude that

$$
\int_{0}^{1}\left|4 \Theta(t)-8 \pi K \log \frac{1}{s}\right| t^{3} d t \leq C .
$$

Proof of Proposition 4.13. First we note that from the upper bound of Proposition 3.1, Lemma 4.4 and Proposition 4.12

$$
2 \pi K \ln \frac{1}{s}+C \geq \mathcal{E}_{\kappa, s}^{L D}\left(\psi_{n, s}, \vec{A}_{s}\right) \geq \mathcal{M}_{\Omega, s}\left(\Psi_{s}, \vec{A}_{s}\right) \geq 2 \pi K \ln \frac{1}{s}-C .
$$

We will usually drop the subscript $s$ in the rest of the proof. Both ends of the sequence of inequalities differ by a constant independent of $s$. Therefore the two 
previous lemmas apply. In particular, from (49) and the first inequality in (52) we find that $t \rightarrow x(t) / s$ is bounded in $L^{1}(0,1-C \sqrt{s})$ independent of $s$. Together with the second inequlity of $(52)$ and the mean value theorem, this implies for each $s$ the existence of $t_{0} \in(1 / 4,3 / 4)$ such that

$$
\frac{x\left(t_{0}\right)}{s} \leq C, \quad\left|\Theta\left(t_{0}\right)-2 \pi K \log \frac{1}{s}\right| \leq C .
$$

Applying Proposition 4.8 with $\omega=\omega_{t_{0}}$ and $\eta=\sigma$ we find balls $\left\{B_{i}\right\}_{1 \leq i \leq m}$ whose sum of radii is less than $\sigma$ such that on each ball (36) holds. We let $U$ be the union of those balls for which $d_{i}$ is different from 0 . There are at most $K$ such balls. Then summing the lower bound (36) for each of the balls in $U$ we find

$$
\Theta_{U}\left(t_{0}\right) \geq 2 \pi K\left(\ln \frac{\sigma}{r\left(\omega_{t_{0}}\right)}-C\right) \geq 2 \pi K \log \frac{1}{s}-C,
$$

using $r\left(\omega_{t_{0}}\right) \leq \mathcal{H}^{1}\left(\partial \omega_{t_{0}}\right)=x\left(t_{0}\right) / f\left(t_{0}\right) \leq C s$. Here we have used the notation

$$
\Theta_{U}\left(t_{0}\right)=\iint_{U \backslash \omega_{t_{0}}}|(\nabla-i \vec{A}) u|^{2}+\iint_{U}(\operatorname{curl} \vec{A})^{2} d x d y .
$$

It follows from (55) and (54) that

$$
\Theta_{T \backslash U}\left(t_{0}\right) \leq C
$$

We claim that this implies

$$
\mathcal{M}_{s, U}(\Psi, A) \geq 2 \pi K \log \frac{1}{s}-C .
$$

First, from Lemma 4.10, applied in $U$ instead of $T$, we have

$$
\mathcal{M}_{s, U}(\Psi, A) \geq \int_{0}^{1} 4 t^{3} \Theta_{U}(t) d t .
$$

Moreover, if $t \geq t_{0}$, then $\omega_{t_{0}} \subset \omega_{t}$. Therefore $\Theta_{T \backslash U}(t) \leq \Theta_{T \backslash U}\left(t_{0}\right)$, and then using (56),

$$
\Theta_{U}(t) \geq \Theta(t)-C \text {. }
$$

On the other hand, if $t<t_{0}$, then $\omega_{t} \subset \omega_{t_{0}}$ and therefore $\Theta_{U}(t) \geq \Theta_{U}\left(t_{0}\right)$ which together with (55) implies

$$
\Theta_{U}(t) \geq 2 \pi K \log \frac{1}{s}-C
$$

Using (58) we find

$$
\mathcal{M}_{s, U}(\Psi, A) \geq \int_{t_{0}}^{1} 4 t^{3} \Theta(t) d t+\int_{0}^{t_{0}} 8 \pi K t^{3} \log \frac{1}{s} d t-C .
$$

Using (52) we find $\mathcal{M}_{s, U}(\Psi, A) \geq \int_{0}^{1} 8 \pi K t^{3} \ln \frac{1}{s} d t-C$ which yields (57).

Now we may conclude the proof of the proposition as follows. From (57) and the energy upper bound, we find

$$
\mathcal{M}_{s, T \backslash U}(\Psi, A) \leq C .
$$

In view of (53) and Lemma 4.4 and the remark following it, this implies that the Lawrence-Doniach energy in $T \backslash U$ is bounded independent of $s$, and from Lemma 4.2 this is also true for the Ginzburg-Landau energy. Thus we have

$$
\iint_{T \backslash \bigcup_{i} B\left(a_{i}, \sigma\right)}\left|\left(\nabla-i \vec{A}_{s}\right) \Psi_{s}\right|^{2}+\left(\operatorname{curl} \vec{A}_{s}\right)^{2} d x d y \leq M(\sigma) .
$$


We are not done though, because our balls depend on $s$. However since their number is bounded by $K$, any sequence $s_{k} \rightarrow 0$ has a subsequence such that the centers of the balls converge, proving the proposition.

\section{Passage to the limit}

In this section we prove Theorem 1.2. For each $s$ we recall that $\Psi(x, y)=\Psi_{s}(x, y)$ (as defined in (23)). Throughout the section we assume that

$$
\left(\psi_{n}, \vec{A}\right) \in t \mathcal{H}_{K}^{*} \text { are minimizers of } \mathcal{E}_{\kappa, s}^{L D} \text {. }
$$

We recall from Lemma 2.2 that this choice of space fixes a gauge for $\left(\psi_{n}, \vec{A}\right)$, and hence energy bounds lead to convergence. From Theorem 4.13 we have a subsequence $s=s_{k} \rightarrow 0$ (for simplicity we will drop the subscript in the following), a finite collection of points $V:=\left\{a_{i}\right\}_{i=1, \ldots, m} \subset \Omega$ with degrees $d_{i}>0$ so that

$$
\begin{aligned}
& \Psi_{s} \rightarrow \Psi_{0} \quad \text { weakly in } H_{l o c}^{1}(\Omega \backslash V), \\
& \vec{A} \rightarrow \vec{A}^{0} \quad \text { weakly in } H^{1}(\Omega),
\end{aligned}
$$

and $\left|\Psi_{0}\right|=1$ almost everywhere in $\Omega \backslash V$.

5.1. The equation for $\Psi_{0}$. We define the "currents" associated with the extended function $\Psi_{s}$,

$$
J_{x}(x, y):=\operatorname{Im}\left\{\Psi_{s}^{*}\left(\partial_{x}-i A_{x}\right) \Psi_{s}\right\}, \quad J_{y}(x, y):=\operatorname{Im}\left\{\Psi_{s}^{*}\left(\partial_{y}-i A_{y}\right) \Psi_{s}\right\} \quad\left(y \neq y_{n}\right),
$$

and denote by $J_{x}^{(n)}, J_{y}^{(n)}$ their restrictions to the strips $y_{n-1}<y<y_{n}$.

Lemma 5.1. Assume (H3). Then

$$
\begin{aligned}
& \text { (61) } J_{y}^{(n)}(x, y)=j_{y}^{(n)}(x), \quad y_{n-1}<y<y_{n}, \\
& (62) \quad J_{x}^{(n)}(x, y)=t(y) j_{x}^{(n)}(x)+(1-t(y)) j_{x}^{(n-1)}(x)+\mathcal{R}_{n}(x, y), \quad y_{n-1} \leq y \leq y_{n},
\end{aligned}
$$

and $\mathcal{R}(x, y)=\mathcal{R}_{n}(x, y), y_{n-1}<y<y_{n}$, satisfies

$$
\|\mathcal{R}\|_{L^{q}(\Omega)} \rightarrow 0 \quad \text { for all } q \in[1,2) .
$$

Proof. The proof of (61) is a straightforward computation. The other identity is more complicated:

$$
\begin{aligned}
J_{x}^{(n)}(x, y):=t^{2} \operatorname{Im} & \left(\psi_{n}^{*}\left(D_{x}^{(n)} \psi_{n}\right)\right)+(1-t)^{2} \operatorname{Im}\left(\psi_{n-1}^{*}\left(D_{x}^{(n-1)} \psi_{n-1}\right)\right) \\
+ & t(1-t) \operatorname{Im}\left(\psi_{n}^{*}\left(D_{x}^{(n-1)} \psi_{n-1}\right) e^{i \int_{y_{n-1}}^{y_{n}} A_{y}(x, y) d y}\right) \\
+ & t(1-t) \operatorname{Im}\left(\psi_{n-1}^{*} e^{-i \int_{y_{n-1}}^{y_{n}} A_{y}\left(x, y_{n-1}\right) d y}\left(D_{x}^{(n)} \psi_{n}\right)\right) \\
& t(1-t) \operatorname{Im}\left(\Psi^{*} i h^{(n)} s\left[\psi_{n}-\psi_{n-1} e^{i \int_{y_{n-1}}^{y_{n}} A_{y}(x, y) d y}\right]\right) .
\end{aligned}
$$

We make the substitutions

$$
\begin{aligned}
& \psi_{n}^{*}\left(D_{x}^{(n-1)} \psi_{n-1}\right) e^{i \int_{y_{n-1}}^{y_{n}} A_{y}(x, y) d y} \\
& =\psi_{n-1}^{*}\left(D_{x}^{(n-1)} \psi_{n-1}\right)+\left(\psi_{n}^{*}-\psi_{n-1}^{*} e^{-i \int_{y_{n-1}}^{y_{n}} A_{y}\left(x, y_{n}\right) d y}\right) \\
& \quad \times\left(D_{x}^{(n-1)} \psi_{n-1}\right) e^{i \int_{y_{n-1}}^{y_{n}} A_{y}(x, y) d y}
\end{aligned}
$$


and

$$
\begin{aligned}
& \psi_{n-1}^{*} e^{-i \int_{y_{n-1}}^{y_{n}} A_{y}\left(x, y_{n}\right) d y}\left(D_{x}^{(n)} \psi_{n}\right) e^{i \int_{y_{n-1}}^{y_{n}} A_{y}(x, y) d y} \\
& \quad=\psi_{n}^{*}\left(D_{x}^{(n)} \psi_{n}\right)-\left(\psi_{n}^{*}-\psi_{n-1}^{*} e^{-i \int_{y_{n-1}}^{y_{n}} A_{y}\left(x, y_{n}\right) d y}\right)\left(D_{x}^{(n)} \psi_{n}\right),
\end{aligned}
$$

to obtain

$$
J_{x}^{(n)}(x, y)=t j_{x}^{(n)}(x)+(1-t) j_{x}^{(n-1)}(x)+\mathcal{R}_{n, 1}-\mathcal{R}_{n, 2},
$$

with

$$
\begin{aligned}
\mathcal{R}_{n, 1}:=t(1-t) \operatorname{Im}( & {\left[\psi_{n}^{*}-\psi_{n-1}^{*} e^{-i \int_{y_{n-1}}^{y_{n}} A_{y} d y}\right] } \\
& \left.\times\left[\left(D_{x}^{(n-1)} \psi_{n-1}\right) e^{i \int_{y_{n-1}}^{y_{n}} A_{y}(x, y) d y}-D_{x}^{(n)} \psi_{n}\right]\right), \\
\mathcal{R}_{n, 2}:=h^{(n)} s \operatorname{Im}\left(i \Psi^{*}\right. & {\left.\left[\psi_{n}-\psi_{n-1} e^{i \int_{y_{n-1}}^{y_{n}} A_{y}(x, y) d y}\right]\right), }
\end{aligned}
$$

for $y_{n-1}<y<y_{n}$.

Now we estimate:

$$
\begin{aligned}
& \int_{y_{n-1}}^{y_{n}} \int_{-L}^{L}\left(\mathcal{R}_{n, 1}\right)^{q} d x d y \\
& \leq s\left(\int_{-L}^{L}\left|\psi_{n}-\psi_{n-1} e^{i \int_{y_{n-1}}^{y_{n}} A_{y}(x, y) d y}\right|^{\frac{2 q}{2-q}}\right)^{\frac{2-q}{2}} \\
& \times\left(\int_{-L}^{L}\left[\left|D_{x}^{(n)} \psi_{n}\right|^{2}+\left|D_{x}^{(n-1)} \psi_{n-1}\right|^{2}\right] d x\right)^{\frac{q}{2}} \\
& \leq 2 s \cdot s^{2-q}\left(\int_{-L}^{L} \frac{1}{s^{2}}\left|\psi_{n}-\psi_{n-1} e^{i \int_{y_{n-1}}^{y_{n}} A_{y}(x, y) d y}\right|^{2}\right)^{\frac{2-q}{2}} \\
& \times\left(\int_{-L}^{L}\left[\left|D_{x}^{(n)} \psi_{n}\right|^{2}+\left|D_{x}^{(n-1)} \psi_{n-1}\right|^{2}\right] d x\right)^{\frac{q}{2}} \\
& \leq C s^{2-q} \cdot s\left(\int_{-L}^{L} \frac{1}{s^{2}}\left|\psi_{n}-\psi_{n-1} e^{i \int_{y_{n-1}}^{y_{n}} A_{y}(x, y) d y}\right|^{2} d x\right. \\
& \left.+\int_{-L}^{L}\left[\left|D_{x}^{(n)} \psi_{n}\right|^{2}+\left|D_{x}^{(n-1)} \psi_{n-1}\right|^{2}\right] d x\right) .
\end{aligned}
$$

Summing over all strips and using periodicity, we obtain

$$
\sum_{n=1-N}^{N}\left\|\mathcal{R}_{n, 1}\right\|_{L^{q}}^{q} \leq C s^{2-q} \mathcal{E}_{\kappa, s}^{L D}\left(\psi_{n}, \vec{A}\right)=o(1) .
$$

Finally,

$$
\begin{aligned}
\sum_{n=1-N}^{N}\left\|\mathcal{R}_{n, 2}\right\|_{L^{2}}^{2} & \leq \sup _{n, x}\left|h^{(n)} s\right|^{2} \int_{-L}^{L} \int_{y_{n-1}}^{y_{n}}\left|\psi_{n}-\psi_{n-1} e^{i \int_{y_{n-1}}^{y_{n}} A_{y}(x, y) d y}\right|^{2} d y d x \\
& \leq C|\ln s|^{2} s^{2} \mathcal{E}_{\kappa, s}^{L D}\left(\psi_{n}, \vec{A}\right)=o(1) .
\end{aligned}
$$

This completes the proof of Lemma 5.1. 
Fix a compact set $B \supset\left\{a_{i}\right\}_{i=1, \ldots, m}$ and choose any $\phi \in C_{0}^{\infty}(\Omega \backslash B)$. With $t=t(y)$ as in (24) we define

(64) $\phi^{(n)}(x):=\frac{1}{s} \int_{y_{n-1}}^{y_{n}} t(y) \phi(x, y) d y, \quad \phi_{(n)}(x):=\frac{1}{s} \int_{y_{n-1}}^{y_{n}}(1-t(y)) \phi(x, y) d y$,

So

$$
\phi^{(n)}(x)+\phi_{(n)}(x)=\frac{1}{s} \int_{y_{n-1}}^{y_{n}} \phi(x, y) d y .
$$

Let $1<q<2$. We have:

$$
\begin{aligned}
& -\int_{-L}^{L} \int_{y_{n-1}}^{y_{n}} J_{x}^{(n)} \partial_{x} \phi d y d x \\
& \quad=-\int_{-L}^{L} \int_{y_{n-1}}^{y_{n}}\left(t j_{x}^{(n)}+(1-t) j_{x}^{(n-1)}\right) \partial_{x} \phi d y d x+o(1)\left\|\partial_{x} \phi\right\|_{L^{\frac{q}{q-1}}} \\
& \quad=\int_{-L}^{L} \int_{y_{n-1}}^{y_{n}}\left(\partial_{x} j_{x}^{(n)} t \phi+\partial_{x} j_{x}^{(n-1)}(1-t) \phi\right) d y d x+o(1) \\
& =\int_{-L}^{L} \int_{y_{n-1}}^{y_{n}}\left[\frac{1}{p}\left(j_{y}^{(n)}-j_{y}^{(n+1)}\right) t \phi\right. \\
& =\int_{-L}^{L}\left[\left(j_{y}^{(n)}-j_{y}^{(n+1)}\right) \phi_{y}^{(n)}(x)+\left(j_{y}^{n-1)}-j_{y}^{(n)}\right) \phi_{(n)}(x)\right] d x+o(1),
\end{aligned}
$$

using the equation of conservation of current (13) in the third step. Since $J_{x}^{(n)}, J_{y}^{(n)}$ are $2 N$-periodic in $n$ we may simplify the expression in summing over $n$ to obtain:

$$
\begin{aligned}
-\sum_{n=1-N}^{N} & \int_{-L}^{L} \int_{y_{n-1}}^{y_{n}} J_{x}^{(n)} \partial_{x} \phi d y d x \\
= & \sum_{n=1-N}^{N} \int_{-L}^{L}\left[\left(j_{y}^{(n)}-j_{y}^{(n+1)}\right) \phi^{(n)}(x)\right. \\
& \left.\quad+\left(j_{y}^{n)}-j_{y}^{(n+1)}\right) \phi_{(n+1)}(x)\right] d x+o(1) \\
= & \sum_{n=1-N}^{N} \int_{-L}^{L} \int_{y_{n-1}}^{y_{n}}\left[\frac{j_{y}^{(n)}-j_{y}^{(n+1)}}{s}\right] \phi(x, y) d x d y \\
& \quad+\sum_{n=1-N}^{N} \int_{-L}^{L}\left[j_{y}^{(n)}-j_{y}^{(n+1)}\right]\left[\phi_{(n+1)}-\phi_{(n)}\right] d x+o(1) .
\end{aligned}
$$


The first term of (65) we sum by parts to obtain:

$$
\begin{aligned}
& \sum_{n=1-N}^{N} \int_{-L}^{L} \int_{y_{n-1}}^{y_{n}}\left[\frac{\left.j_{y}^{(n)}-j_{y}^{(n+1)}\right]}{s}\right] \phi(x, y) d x d y \\
& \quad=\sum_{n=1-N}^{N} \int_{-L}^{L} \int_{y_{n-1}}^{y_{n}}\left[\frac{J_{y}^{(n)}-J_{y}^{(n+1)}}{s}\right] \phi(x, y) d x d y \\
& =\sum_{n=1-N}^{N} \int_{-L}^{L} \int_{y_{n-1}}^{y_{n}} J_{y}^{(n)}\left[\frac{\phi(x, y)-\phi(x, y-s)}{s}\right] d x d y \\
& =\sum_{n=1-N}^{N} \int_{-L}^{L} \int_{y_{n-1}}^{y_{n}} J_{y}^{(n)} \partial_{y} \phi(x, y) d x d y+o(1),
\end{aligned}
$$

since supp $\phi \subset \subset \Omega \backslash B$, and $\Psi \rightarrow \Psi_{0}, \vec{A} \rightarrow \vec{A}^{0}$ weakly in $H^{1}(\Omega \backslash B)$.

To estimate the last term in (65) we sum by parts: let $\epsilon>0$. Then,

$$
\begin{aligned}
\left|\sum_{n=1-N}^{N} \int_{-L}^{L}\left[j_{y}^{(n)}-j_{y}^{(n+1)}\right]\left[\phi_{(n+1)}-\phi_{(n)}\right] d x\right| & \left|\sum_{n=1-N}^{N} \int_{-L}^{L} j_{y}^{(n)}\left[\phi_{(n+1)}-2 \phi_{(n)}+\phi_{(n-1)}\right] d x\right| \\
= & \mid \sum_{n=1-N}^{N} \int_{-L}^{L} \int_{y_{n-1}}^{y_{n}} j_{y}^{(n)}(1-t(y)) \\
& \times\left[\frac{\phi(x, y+s)-2 \phi(x, y)+\phi(x, y-s)}{s}\right] d x \mid \\
\leq & \frac{\epsilon}{2} \sum_{n=1-N}^{N} \int_{-L}^{L} \int_{y_{n-1}}^{y_{n}}\left(j_{y}^{(n)}\right)^{2} d y d x \\
& +\frac{1}{2 \epsilon} \sum_{n=1-N}^{N} \int_{-L}^{L} \int_{y_{n-1}}^{y_{n}}\left[\frac{\phi(x, y+s)-2 \phi(x, y)+\phi(x, y-s)}{s}\right]^{2} d x \\
\leq & \frac{\epsilon s}{2} \sum_{n=1-N}^{N} \int_{-L}^{L} \frac{1}{s^{2}}\left|\psi_{n}-\psi_{n-1} e^{i \int_{y_{n}-1}^{y_{n}} A_{y}(x, y) d y}\right|^{2} d x \\
& +\frac{s^{2}}{\epsilon} \sum_{n=1-N}^{N} \int_{-L}^{L} \int_{y_{n-1}}^{y_{n}}\left[\frac{\phi(x, y+s)-2 \phi(x, y)+\phi(x, y-s)}{s^{2}} d x\right. \\
\leq & \mathcal{E}_{\kappa, s}^{L D}\left(\psi_{n}, \vec{A}\right)+\frac{s^{2}}{\epsilon} C\left\|^{2} \phi\right\|_{H^{2}(\Omega)} \cdot
\end{aligned}
$$

Taking $\epsilon=s$ for example, we see that this term is negligible as $s \rightarrow 0$ and hence (65) gives

$$
\iint_{\Omega}\left(J_{0, x} \partial_{x} \phi+J_{0, y} \partial_{y} \phi\right) d x d y=0
$$

for all $\phi \in C_{0}^{\infty}(\Omega \backslash B)$, where $B$ is any closed neigborhood of the vortices $\left\{a_{1}, \ldots, a_{m}\right\}$. In other words, $\overrightarrow{J_{0}}$ is a weak solution of $\operatorname{div} \overrightarrow{J_{0}}=0$ in $\Omega \backslash V$. As $\operatorname{div} \vec{A}^{0}=0$ we 
conclude that $\Psi_{0}$ is a weak solution of

$$
\operatorname{div}\left(\operatorname{Im}\left\{\Psi_{0}^{*} \nabla \Psi_{0}\right\}\right)=0
$$

with $\left|\Psi_{0}\right|=1$ in $\Omega \backslash V$. From [BeRi] we conclude that $\Psi_{0}$ is a (weak) harmonic map in $\Omega \backslash V$. (For the smoothness of $\Psi_{0}$, see Remark 5.5 below.) Therefore we have proven all of part (a) of Theorem 1.2 except for the statement about the degree (which will follow from the result of the third subsection).

\subsection{Local compactness for $h$.}

Lemma 5.2. Under the same hypotheses as Theorem 1.2, along some subsequence $s \rightarrow 0, h_{s}(x, y) \rightarrow h_{0}(x, y)$ in $L_{l o c}^{2}(\Omega \backslash V)$ strongly, and $h_{0} \in H_{l o c}^{1}(\Omega \backslash V)$.

Let $\delta>0$ be given and let

$$
\Omega_{\delta}:=\Omega \backslash \bigcup_{i=1}^{m} B_{\delta}\left(a_{i}\right), \quad \Omega_{4 \delta}:=\Omega \backslash \bigcup_{i=1}^{m} B_{4 \delta}\left(a_{i}\right) .
$$

From Proposition 4.13 there exists a constant $C_{1}=C_{1}(\delta)$ so that

$$
\mathcal{E}_{\kappa, s}^{L D}\left(\psi_{n}, \vec{A} ; \Omega_{4 \delta}\right) \leq \mathcal{E}_{\kappa, s}^{L D}\left(\psi_{n}, \vec{A} ; \Omega_{\delta}\right) \leq C_{1} .
$$

For any $(u, v) \in \mathbf{R}^{2}$ with $|(u, v)|<\delta$, we estimate the following difference:

$$
\begin{aligned}
& \|h(x+u, y+v)-h(x, y)\|_{L^{2}\left(\Omega_{4 \delta}\right)} \leq\|h(x+u, y+v)-h(x, y+v)\|_{L^{2}\left(\Omega_{4 \delta}\right)} \\
& \|h(x, y+v)-h(x, y)\|_{L^{2}(4 \delta)} .
\end{aligned}
$$

First, assume that $y_{n-1}<y+v \leq y_{n}$ and denote $\Omega_{4 \delta, n}:=\Omega_{4 \delta} \cap\left\{y_{n-1}<y \leq y_{n}\right\}$. Then,

$$
\begin{aligned}
\iint_{\Omega_{4 \delta, n}} & |h(x+u, y+v)-h(x, y+v)|^{2} d x d y \\
= & \iint_{\Omega_{4 \delta, n}}\left|h^{(n)}(x+u)-h^{(n)}(x)\right|^{2} d x d y \\
\leq & u^{2} \iint_{\Omega_{4 \delta, n}}\left|\partial_{x} h^{(n)}\right|^{2} d x d y \\
= & \frac{u^{2}}{s^{2}} \iint_{\Omega_{4 \delta, n}}\left|\psi_{n}-\psi_{n-1} e^{i \int_{y_{n-1}}^{y_{n}} A_{y}(x, y) d y}\right|^{2} d x d y .
\end{aligned}
$$

Summing over $n$, we obtain:

$$
\begin{aligned}
& \iint_{\Omega_{4 \delta}}|h(x+u, y+v)-h(x, y+v)|^{2} d x d y \\
& \quad \leq u^{2} \sum_{1-N}^{N} \iint_{\Omega_{4 \delta, n}} \frac{1}{s^{2}}\left|\psi_{n}-\psi_{n-1} e^{i \int_{y_{n-1}}^{y_{n}} A_{y}(x, y) d y}\right|^{2} d x d y \\
& \quad \leq u^{2} \mathcal{E}_{\kappa, s}^{L D}\left(\psi_{n}, \vec{A} ; \Omega_{4 \delta}\right) \leq C_{1} u^{2},
\end{aligned}
$$

by $(70)$.

For the second term, we write

$$
v=n_{0} s+r, \quad n_{0} \in \mathbf{Z}, 0 \leq r<s .
$$


Thus, when $y \in\left(y_{n-1}, y_{n}\right)$ we have

$$
h(x, y+v)= \begin{cases}h^{\left(n+n_{0}\right)}(x), & \text { when } y_{n-1}<y<y_{n}-r, \\ h^{\left(n+n_{0}+1\right)}(x), & \text { when } y_{n}-r<y<y_{n} .\end{cases}
$$

In the case $y_{n-1}<y<y_{n}-r$ we have

$$
h(x, y+v)-h(x, y)=\sum_{k=n}^{n+n_{0}-1}(x)\left(h^{(k+1)}(x)-h^{(k)}(x)\right),
$$

and hence

$$
\begin{aligned}
& \iint_{\Omega_{4 \delta} \cap\left\{y_{n-1}<y<y_{n}-r\right\}}[h(x, y+v)-h(x, y)]^{2} d x d y \\
& \quad \leq \iint_{\Omega_{4 \delta} \cap\left\{y_{n-1}<y<y_{n}-r\right\}}\left[\sum_{k=n}^{n+n_{0}-1}\left(h^{(k+1)}(x)-h^{(k)}(x)\right)\right]^{2} d x d y \\
& \quad \leq n_{0} \iint_{\Omega_{4 \delta} \cap\left\{y_{n-1}<y<y_{n}-r\right\}} \sum_{k=n}^{n+n_{0}-1}\left(h^{(k+1)}(x)-h^{(k)}(x)\right)^{2} d x d y \\
& \leq n_{0} s^{2} \iint_{\Omega_{4 \delta} \cap\left\{y_{n-1}<y<y_{n}-r\right\}} \sum_{k=n}^{n+n_{0}-1} \mid\left(\nabla-\left.i A_{x}\left(x, y_{k}\right) \psi_{k}\right|^{2} d x d y .\right.
\end{aligned}
$$

Similarly for $y_{n}-r<y<y_{n}$, we obtain

$$
\begin{aligned}
& \iint_{\Omega_{4 \delta} \cap\left\{y_{n}-r<y<y_{n}\right\}}[h(x, y+v)-h(x, y)]^{2} d x d y \\
& \quad \leq\left(n_{0}+1\right) s^{2} \iint_{\Omega_{4 \delta} \cap\left\{y_{n}-r<y<y_{n}\right\}} \sum_{k=n}^{n+n_{0}}\left|\left(\nabla-i A_{x}\left(x, y_{k}\right)\right) \psi_{k}\right|^{2} d x d y .
\end{aligned}
$$

To conclude we note that if $|(u, v)|<\delta$ and $(x, y) \in \Omega_{4 \delta}$ with $y_{n-1}<y<y_{n}$, then the points $\left(x, y_{k}\right) \in \Omega_{\delta}$ for $k=n, \ldots, n+n_{0}+1$. Hence we combine (72) and (73) together to obtain:

$$
\begin{aligned}
& \iint_{\Omega_{4 \delta}}(h(x, y+v)-h(x, y))^{2} d x d y \\
& \quad \leq \sum_{n=1-N}^{N}\left(n_{0}+1\right) s^{2} \sum_{k=n}^{n+n_{0}} \iint_{\Omega_{4 \delta, k}}\left|\left(\nabla-i A_{x}\left(x, y_{k}\right)\right) \psi_{k}\right|^{2} d x d y \\
& \quad \leq\left(n_{0}+1\right)^{2} s^{2} \sum_{n=1-N}^{N} \iint_{\Omega_{\delta} \cap\left\{y_{n-1}<y<y_{n}\right\}}\left|\left(\nabla-i A_{x}\left(x, y_{n}\right)\right) \psi_{n}\right|^{2} d x d y \\
& \leq(v+s)^{2} \mathcal{E}_{\kappa, s}^{L D}\left(\psi_{n}, \vec{A} ; \Omega_{\delta}\right) \leq C_{1}(v+s)^{2} .
\end{aligned}
$$

From (71) and (74) we see that

$$
\|h(x+u, y+v)-h(x, y)\|_{L^{2}\left(\Omega_{4 \delta}\right)} \leq C_{1}\left(u^{2}+(v+s)^{2}\right) .
$$


By Théorem IV.25 of Brezis [Br] and a diagonal argument, for any sequence $s \rightarrow 0$ we may extract a subsequence such that $h=h_{s} \rightarrow h_{0}$ in $L^{2}\left(\Omega_{4 \delta}\right)$ with $h_{0} \in L^{2}\left(\Omega_{4 \delta}\right)$. Passing to the limit $s \rightarrow 0$ in (75) along this subsequence we obtain the analogous inequality for $h_{0}$,

$$
\left\|h_{0}(x+u, y+v)-h_{0}(x, y)\right\|_{L^{2}\left(\Omega_{4 \delta}\right)} \leq C_{1}\left(u^{2}+v^{2}\right)
$$

which implies that $h_{0} \in H^{1}\left(\Omega_{4 \delta}\right)$ by Proposition IX.3 (iii) of [Br]. Since $\delta>0$ is arbitrary, this proves Lemma 5.2.

We define

$$
\left(\delta_{s} h\right)(x, y):=\frac{h^{(n+1)}(x)-h^{(n)}(x)}{s}, \quad y_{n-1}<y<y_{n}
$$

Lemma 5.3. Under the same hypotheses as Lemma 5.2,

$$
\delta_{s} h \rightarrow \frac{\partial h_{0}}{\partial y}, \quad \frac{\partial h}{\partial x} \rightarrow \frac{\partial h_{0}}{\partial x} \quad \text { weakly in } L_{l o c}^{2}(\Omega \backslash V)
$$

Proof. Take $\zeta \in C_{0}^{\infty}(\Omega \backslash V)$. By partial summation,

$$
\begin{aligned}
\iint_{\Omega}\left(\delta_{s} h\right) \zeta d x d y & =\sum_{n=1-N}^{N} \int_{-L}^{L} \int_{y_{n-1}}^{y_{n}} h^{(n)}(x)\left(\frac{\zeta(x, y-s)-\zeta(x, y)}{s}\right) d y d x \\
& \rightarrow-\iint_{\Omega} h_{0} \frac{\partial \zeta}{\partial y} d x d y
\end{aligned}
$$

by Lemma 5.2. So $\delta_{s} h \rightarrow h_{0}$ in the sense of distributions on $\Omega \backslash V$. But we already know $h_{0} \in H_{l o c}^{1}(\Omega \backslash V)$, and so the integration by parts is justified. The proof that $\frac{\partial h}{\partial x} \rightarrow \frac{\partial h_{0}}{\partial x}$ is even simpler, and is omitted.

5.3. The equation for the magnetic field. We are now ready to find the limiting equation for $h_{0}$.

Proposition 5.4. $h_{0}$ is smooth in $\Omega \backslash V$ and $-\Delta h_{0}+h_{0}=0$ in $\Omega \backslash V$.

Proof. Let $\phi \in C_{0}^{\infty}(\Omega \backslash V)$. With $\phi^{(n)}(x)$ defined as in (64) we calculate:

$$
\begin{aligned}
\int_{y_{n-1}}^{y_{n}} t(y) j_{x}^{(n)} \partial_{y} \phi d y & =j_{x}^{(n)}(x)\left[\phi\left(x, y_{n}\right)-\phi^{(n)}(x)\right], \quad \text { and } \\
\int_{y_{n-1}}^{y_{n}}(1-t(y)) j_{x}^{(n-1)} \partial_{y} \phi d y & =j_{x}^{(n-1)}(x)\left[-\phi\left(x, y_{n-1}\right)+\phi^{(n)}(x)\right] .
\end{aligned}
$$


By partial summation using the Euler-Lagrange equation (10) we have

$$
\begin{aligned}
& \iint_{\Omega} J_{x} \partial_{y} \phi d x d y \\
& =\sum_{n=1-N}^{N} \int_{-L}^{L} \int_{y_{n-1}}^{y_{n}}\left[t(y) j_{x}^{(n)}+(1-t) j_{x}^{(n-1)}\right] \partial_{y} \phi d x d y+o(1) \\
& =\sum_{n=1-N}^{N} \int_{-L}^{L}\left\{j_{x}^{(n)}\left[\phi\left(x, y_{n}\right)-\phi^{(n)}\right]+j_{x}^{(n-1)}\left[\phi^{(n)}-\phi\left(x, y_{n-1}\right)\right]\right\} d x+o(1) \\
& =\sum_{n=1-N}^{N} \int_{-L}^{L}\left[j_{x}^{(n)} \phi\left(x, y_{n}\right)-j_{x}^{(n-1)} \phi\left(x, y_{n-1}\right)\right] d x \\
& -\sum_{n=1-N}^{N} \int_{-L}^{L}\left[j_{x}^{(n)}-j_{x}^{(n-1)}\right] \phi^{(n)} d x+o(1) \\
& =\sum_{n=1-N}^{N} \int_{-L}^{L} j_{x}^{(n)}\left[\phi^{(n+1)}-\phi^{(n)}\right] d x+o(1) \\
& =\sum_{n=1-N}^{N} \int_{-L}^{L} \int_{y_{n-1}}^{y_{n}} \frac{h^{(n+1)}-h^{(n)}}{s} \cdot \frac{\phi(x, y+s)-\phi(x, y)}{s} d x d y+o(1) \\
& =\iint_{\Omega}\left(\delta_{s} h\right) \frac{\phi(x, y+s)-\phi(x, y)}{s} d x d y+o(1) \\
& =\iint_{\Omega} \partial_{y} h_{0} \cdot \partial_{y} \phi(x, y) d x d y+o(1),
\end{aligned}
$$

since $\delta_{s} h \rightarrow \partial_{y} h_{0}$ in $L^{2}(\Omega \backslash V)$ by Lemma 5.3 , and $\frac{\phi(x, y+s)-\phi(x, y)}{s} \rightarrow \partial_{y} \phi(x, y)$ in $L^{2}(\Omega)$. (Note that in the third line, the sum is telescopic, and hence vanishes by periodicity.)

On the other hand, by (11), (61), and Lemma 5.3,

$$
\begin{aligned}
\iint_{\Omega} J_{y}(x, y) \partial_{x} \phi(x, y) d x d y & =\sum_{n=1-N}^{N} \int_{-L}^{L} \int_{y_{n-1}}^{y_{n}} j_{y}^{(n)} \partial_{x} \phi(x, y) d y d x \\
& =\sum_{n=1-N}^{N} \int_{-L}^{L} \int_{y_{n-1}}^{y_{n}} \partial_{x} h^{(n)} \partial_{x} \phi(x, y) d y d x \\
& =\iint_{\Omega} \partial_{x} h_{0}(x, y) \partial_{x} \phi(x, y) d x d y+o(1)
\end{aligned}
$$

With (77) we have

$$
\iint_{\Omega} \nabla h_{0} \cdot \nabla \phi d x d y=\lim _{s \rightarrow 0} \iint_{\Omega}\left[J_{x} \partial_{y} \phi-J_{y} \partial_{x} \phi\right] d x d y .
$$


Now, we already have $\Psi \rightarrow \Psi_{0}$ and $\vec{A} \rightarrow \vec{A}^{0}$ weakly in $H_{\text {loc }}^{1}(\Omega \backslash V)$. Therefore the associated currents

$$
J_{x} \rightarrow J_{0, x}:=\operatorname{Im}\left\{\Psi_{0}^{*}\left(\partial_{x}-i A_{x}^{0}\right) \Psi_{0}\right\}, \quad J_{y} \rightarrow J_{0, y}:=\operatorname{Im}\left\{\Psi_{0}^{*}\left(\partial_{y}-i A_{y}^{0}\right) \Psi_{0}\right\}
$$

weakly in $L^{q}(\Omega \backslash V)$ for $1<q<2$. Moreover, since $\left|\Psi_{0}\right|=1$ almost everywhere in $\Omega \backslash V$ we can write $\Psi_{0}=e^{i \chi}$ with $\chi \in H_{l o c}^{1}(\Omega \backslash V)$, and $\overrightarrow{J_{0}}=\nabla \chi-\vec{A}^{0}$. Returning to $(78)$, we substitute and integrate by parts,

$$
\begin{aligned}
\iint_{\Omega} \nabla h_{0} \cdot \nabla \phi d x d y & =\lim _{s \rightarrow 0} \iint_{\Omega}\left[J_{x} \partial_{y} \phi-J_{y} \partial_{x} \phi\right] d x d y \\
& =-\iint_{\Omega} \overrightarrow{J_{0}} \cdot \nabla^{\perp} \phi d x d y \\
& =-\iint_{\Omega}\left(\nabla \chi-\vec{A}^{0}\right) \cdot \nabla^{\perp} \phi d x d y \\
& =-\iint_{\Omega}\left(\nabla \times \vec{A}^{0}\right) \phi d x d y=-\iint_{\Omega} h_{0} \phi d x d y .
\end{aligned}
$$

In particular, $-\Delta h_{0}+h_{0}=0$ in the sense of distributions on $\Omega \backslash V$. By standard regularity theory $h_{0} \in C^{\infty}(\Omega \backslash V)$, and the equation holds pointwise in $\Omega \backslash V$.

Remark 5.5. Since we can pass to the weak limit in the Euler-Lagrange equations (12) and (11) we have

$$
\nabla^{\perp} h_{0}(x, y)=-\vec{J}_{0}(x, y), \quad \text { for all }(x, y) \notin V
$$

and hence $\overrightarrow{J_{0}} \in C^{\infty}(\Omega \backslash V)$ as well. Furthermore, from our choice of gauge (see (7)) we may also conclude $\vec{A}^{0} \in C^{\infty}(\Omega \backslash V)$. In particular, the phase $\chi \in C^{\infty}(\Omega \backslash V)$ and therefore $\Psi_{0}=e^{i \chi} \in C^{\infty}(\Omega \backslash V)$ as well.

Proposition 5.6. $-\Delta h_{0}+h_{0}=2 \pi \sum_{i=1}^{K} \delta_{a_{i}}$ in the sense of distributions on $\Omega$.

Proof. We follow Serfaty [Se]. From the previous lemma, $-\Delta h_{0}+h_{0}$ is a distribution with support on the finite point set $V=\left\{a_{1}, \ldots, a_{m}\right\}$. Therefore there exist coefficients $c_{i, \alpha}$ for $i=1, \ldots, m$ and multi-indices $\alpha$ so that

$$
-\Delta h_{0}+h_{0}=\sum_{i, \alpha} c_{i, \alpha} \partial^{\alpha} \delta_{a_{i}}
$$

But $h_{0} \in L^{2}(\Omega)$ so $-\Delta h_{0}+h_{0} \in W^{-2,2}(\Omega)$, and therefore we must have

$$
-\Delta h_{0}+h_{0}=\sum_{i} c_{i} \delta_{a_{i}}
$$

and it suffices to calculate the coefficients $a_{i}$.

For any $\delta>0$ sufficiently small such that $3 \delta<\operatorname{dist}\left(a_{i}, a_{j}\right)$ for all $i \neq j$, take a test function $\zeta \in C_{0}^{\infty}(\Omega)$ with $\zeta(x, y) \equiv 1$ for $(x, y) \in B_{2 \delta}\left(a_{j}\right)$ for some $j$ and $\zeta(x, y) \equiv 0$ for $(x, y) \in B_{\delta}\left(a_{i}\right)$ for all $i \neq j$. Then, twice integrating by parts away 
from the vortices $\left\{a_{i}\right\}$ and applying (79),

$$
\begin{aligned}
c_{j} & =\iint_{\Omega} h_{0}(-\Delta \zeta+\zeta) d x d y \\
& =\iint_{B_{\delta}\left(a_{j}\right)} h_{0} d x d y+\iint_{\Omega \backslash\left[\cup_{i} B_{\delta}\left(a_{j}\right)\right]} h_{0}(-\Delta \zeta+\zeta) d x d y \\
& =\iint_{B_{\delta}\left(a_{j}\right)} h_{0} d x d y+\iint_{\Omega \backslash\left[\cup_{i} B_{\delta}\left(a_{j}\right)\right]}\left[-\Delta h_{0}+h_{0}\right] \zeta d x d y-\int_{\partial B_{\delta}\left(a_{j}\right)} \nabla h_{0} \cdot \vec{\nu} d s \\
& =\iint_{B_{\delta}\left(a_{j}\right)} h_{0} d x d y+\int_{\partial B_{\delta}\left(a_{j}\right)} \vec{J}_{0} \cdot \vec{\tau} d s \\
& =\iint_{B_{\delta}\left(a_{j}\right)} h_{0} d x d y+\int_{\partial B_{\delta}\left(a_{j}\right)} \frac{\partial \chi}{\partial s} d s-\int_{\partial B_{\delta}\left(a_{j}\right)} \vec{A}^{0} \cdot d \vec{s} \\
& =2 \pi \operatorname{deg}\left(\Psi_{0}, \partial B_{\delta}\left(a_{j}\right)\right) .
\end{aligned}
$$

We now show that the degree of $\Psi_{0}$ at each vortex is preserved in the weak $H^{1}$ limit, and hence $c_{j}=d_{j}, j=1, \ldots, m$. Let $\delta>0$ be given. First, we claim that there exists $r \in(\delta, 2 \delta), M>0$, and a subsequence $s_{n} \rightarrow 0$ such that

$$
\int_{\partial B_{r}\left(a_{i}\right)}\left|\nabla_{A} \Psi_{s_{n}}\right|^{2} d s \leq M
$$

Let $f_{s}(r):=\int_{\partial B_{r}\left(a_{i}\right)}\left|\nabla_{A} \Psi_{s}\right|^{2} d s$, and assume the contrary: for every $M>0$ and for every $r \in(\delta, 2 \delta)$ there exists $s_{0}=s_{0}(r)$ such that $f_{s}(r)>M$ for all $s \leq s_{0}(r)$. In particular, $\liminf _{s \rightarrow 0} f_{s}(r) \geq M$ for each $r \in(\delta, 2 \delta)$, and so by Fatou's Lemma and Fubini's Theorem we conclude that

$$
\begin{aligned}
\delta M & \leq \int_{\delta}^{2 \delta} \liminf _{s \rightarrow 0} f_{s}(r) d r \\
& \leq \liminf _{s \rightarrow 0} \int_{\delta}^{2 \delta} f_{s}(r) d r=\liminf _{s \rightarrow 0} \int_{B_{2 r}\left(a_{i}\right) \backslash B_{r}\left(a_{i}\right)}\left|\nabla_{A} \Psi_{s}\right|^{2} d x
\end{aligned}
$$

The right-hand side is uniformly bounded for each fixed $\delta$ by Proposition 4.13, but $M$ is arbitrary, giving the desired contradiction and proving (80).

From (80), Proposition 4.13 and the trace inequality applied to $A$, we have

$$
\left\|\nabla \Psi_{s_{n}}\right\|_{L^{2}\left(\partial B_{r}\left(a_{i}\right)\right)} \leq\left\|\nabla_{A} \Psi_{s_{n}}\right\|_{L^{2}\left(\partial B_{r}\left(a_{i}\right)\right)}+\|A\|_{L^{2}\left(\partial B_{r}\left(a_{i}\right)\right)} \leq C
$$

Since $\left|\Psi_{s}(x)\right| \leq 1$ a.e., we conclude that a further subsequence of $\Psi_{s_{n}} \rightarrow \Psi_{0}$ weakly in $H^{1}\left(\partial B_{r}\left(a_{i}\right)\right)$, and therefore the degree is preserved in the limit, $\operatorname{deg}\left(\Psi_{0}, \partial B_{r}\left(a_{i}\right)\right)$ $=\operatorname{deg}\left(\Psi_{s_{n}}, \partial B_{r}\left(a_{i}\right)\right)=d_{i}$.

It remains to show that $d_{i}=1$ for all $i$. From Lemma 1 of $[\mathrm{S}]$ there exists a constant $C_{1}$ independent of $s, \delta$ such that

$$
\iint_{\Omega \backslash \bigcup_{i} B_{\delta}\left(a_{i}\right)}\left\{\left|(\nabla-i \vec{A}) \Psi_{0}\right|^{2}+h^{2}\right\} d x d y \geq 2 \pi \sum_{i=1}^{m} d_{i}^{2}|\ln \delta|-C_{1} .
$$


In particular, by weak convergence

$$
\begin{aligned}
& \liminf _{s \rightarrow 0} \iint_{\Omega \backslash \bigcup_{i} B_{\delta}\left(a_{i}\right)}\left\{\left|(\nabla-i \vec{A}) \Psi_{s}\right|^{2}+h^{2}\right\} d x d y \\
& \geq \iint_{\Omega \backslash \bigcup_{i} B_{\delta}\left(a_{i}\right)}\left\{\left|(\nabla-i \vec{A}) \Psi_{0}\right|^{2}+h^{2}\right\} d x d y \\
& \quad \geq 2 \pi \sum_{i=1}^{m} d_{i}^{2}|\ln \delta|-C_{1} .
\end{aligned}
$$

Furthermore, from the construction of the vortex balls (36) there is another constant $C_{2}$, also independent of $\delta, s$ such that

$$
\iint_{\bigcup_{i} B_{\delta}\left(a_{i}\right)}\left\{\left|(\nabla-i \vec{A}) \Psi_{s}\right|^{2}+h^{2}\right\} d x d y \geq 2 \pi \sum_{i=1}^{m}\left|d_{i}\right| \ln \left[\frac{\delta}{s}\right]-C_{2} .
$$

Applying the upper and lower bounds on the energy,

$$
\begin{aligned}
2 \pi K|\ln s|+O(1) & \geq \mathcal{E}_{\kappa, s}^{L D}\left(\psi_{n}, \vec{A}\right) \\
& \geq \iint_{\Omega}\left\{\left|(\nabla-i \vec{A}) \Psi_{s}\right|^{2}+h^{2}\right\} d x d y \\
& \geq 2 \pi \sum_{i=1}^{m}\left\{d_{i}^{2}|\ln \delta|+\left|d_{i}\right| \ln \left[\frac{\delta}{s}\right]\right\}-O(1) \\
& =2 \pi \sum_{i=1}^{m}\left|d_{i}\right||\ln s|+2 \pi \sum_{i=1}^{m}\left(d_{i}^{2}-\left|d_{i}\right|\right)|\ln \delta|+O(1) .
\end{aligned}
$$

In particular, we must have either $d_{i}=0$ or $d_{i}=1$ for all $i=1, \ldots, m$. However, in the construction of the balls in Proposition 4.13 we have eliminated balls with degree $d_{i}=0$, and therefore we conclude that $d_{i}=1$ for all $i=1, \ldots, m=K$. This concludes the proof of (a) and (b) in Theorem 1.2. To obtain (c) we combine the upper bound in Proposition 3.1 with the lower bounds of Lemma 4.4 and Proposition 4.12.

\section{ACKNOWLEDGEMENT}

The first and second authors wish to thank J. Berlinsky for introducing them to this problem and for his helpful discussions on the physical background.

\section{REFERENCES}

[ABeB1] S. Alama, L. Bronsard, and A.J. Berlinsky, Periodic vortex lattices for the LawrenceDoniach model of layered superconductors in a parallel field, Commun. Contemp. Math., vol. 3 (2001), no. 3, 457-494. MR1849651 (2003b:82067)

[ABeB2] S. Alama, L. Bronsard, and A.J. Berlinsky, Minimizers of the Lawrence-Doniach energy in the small-coupling limit: finite width samples in a parallel field, Annales IHP-Analyse nonlinéaire, vol. 19, (2002), 281-312. MR1956952 (2003m:82102)

[An] P.W. Anderson, c-Axis Electrodynamics as Evidence for the Interlayer Theory of HighTemperature Superconductivity, Science, vol. 279 (1998), pp. 1196-1198.

[BaK] P. Bauman, Y. Ko, Analysis of solutions to a coupled Ginzburg-Landau system for layered superconductors, preprint.

[BBH] F. Bethuel, H. Brezis, F. Hélein, "Ginzburg-Landau Vortices". Birkhäuser, Boston, 1994. MR1269538 (95c:58044) 
[BeRi] F. Bethuel, T. Rivière, Vortices for a variational problem related to superconductivity, Ann. Inst. Henri Poincaré, Analyse non linéaire, vol. 12 (1995), 243-303. MR1340265 (96g:35045)

[Bo] R. Bott, L. Tu, "Differential forms in algebraic topology." Springer, New York, 1982. MR0658304 (83i:57016)

[Br] H. Brezis, "Analyse fonctionelle. Théorie et applications." Masson, Paris, 1983. MR0697382 (85a:46001)

[Bu] L. Bulaevskiǔ, Magnetic properties of layered superconductors with weak interaction between the layers, Sov. Phys. JETP, vol. 37 (1973), pp 1133-1136.

[BuCm] L. Bulaevskiǔ and J. Clem, Vortex lattice of highly anisotropic layered superconductors in strong, parallel magnetic fields, Phys. Rev. B44 (1991), pp 10234-10238.

[ChDG] S.J. Chapman, Q. Du, M. Gunzburger, On the Lawrence-Doniach and anisotropic Ginzburg-Landau models for layered superconductors, SIAM J. Appl. Math., vol. 55 (1995), pp. 156-174. MR1313011 (95m:82120)

[CmCo] J. Clem and M. Coffey, Viscous flux motion in a Josephson-coupled layer model of high-T $T_{c}$ superconductors, Phys. Rev. vol. B42 (1990), pp. 6209-6216.

[Fa] B. Farid, Rosencrantz and Guildenstern may not be dead; on the interlayer vortices in Tl-2201, J. Phys., vol. C 10 (1998), pp. L589-L596.

[Iy] Y. Iye, How Anisotropic Are the Cuprate High $T_{c}$ Superconductors? Comments Cond. Mat. Phys., vol. 16 (1992), pp. 89-111.

[Je] R. Jerrard, Lower bounds for generalized Ginzburg-Landau functionals, SIAM Jour. Math. Anal., vol. 30 (1999), pp. 721-746. MR1684723 (2001f:35115)

[KAVB] P. Kes, J. Aarts, V. Vinokur, and C. van der Beek, Dissipation in Highly Anisotropic Superconductors, Phys. Rev. Lett. vol. 64 (1990), pp 1063-1066.

[LaDo] W. Lawrence and S. Doniach, Proceedings of the Twelfth International Conference on Low Temperature Physics, E. Kanda (ed.), Academic Press of Japan, Kyoto, 1971, p. 361 .

[Mo] K.A. Moler, J.R. Kirtley, D.G. Hinks, T.W. Li, and M. Xu, Images of Interlayer Josephson Vortices in $\mathrm{Tl}_{2} \mathrm{Ba}_{2} \mathrm{CuO}_{6+\delta}$, Science, vol. 279 (1998), pp. 1193-1196.

[S] E. Sandier, Lower Bounds for the Energy of Unit Vector Fields and Applications, J. Functional Analysis, vol. 152 (1998), 379-403.

[Se] S. Serfaty, Local Minimizers for the Ginzburg-Landau Energy near Critical Magnetic Field, Part I, Comm. Contemp. Math., vol. 1 (1999), 213-254. MR1696100 (2001a:82077)

[tH] G. $\mathrm{t}$ 'Hooft, A property of electric and magnetic flux in non-Abelian gauge theories, Nucl. Phys., Vol. B153 (1979), pp. 141-160. MR0535106 (80g:81043)

Department of Mathematics and Statistics, McMaster University, Hamilton, OnTARIO, CANADA L8S 4K1

Department of Mathematics and Statistics, McMaster University, Hamilton, OnTARIO, CANADA L8S $4 \mathrm{~K} 1$

Departement des Mathématiques, Université Paris XiI, 64 aVenue du Général de Gaulle, 94010 Créteil Cedex, France 